\title{
Different analysis strategies of 16S rRNA gene data from rodent studies generate contrasting views of gut bacterial communities associated with diet, health and obesity
}

\author{
Jose F Garcia-Mazcorro \\ Noratto ${ }^{5}$ \\ ${ }^{1}$ Research and Development, MNA de Mexico, San Nicolas de los Garza, Nuevo Leon, Mexico \\ Faculty of Agronomy, Universidad Autónoma de Nuevo León, General Escobedo, Nuevo Leon, Mexico \\ 3 School of Engineering and Sciences, Tecnologico de Monterrey, Monterrey, Nuevo Leon, Mexico \\ 4 Department of Nutrition and Food Sciences, Texas A\&M University, College Station, Texas, United States \\ 5 Department of Food Science and Technology, Texas A\&M University, College Station, Texas, United States \\ Corresponding Author: Jose F Garcia-Mazcorro \\ Email address: josegarcia_mex@hotmail.com
}

, Jorge R. Kawas ${ }^{2}$, Cuauhtemoc Licona Cassani ${ }^{3}$, Susanne Mertens-Talcott ${ }^{4}$, Giuliana

Background. One of the main functions of diet is to nurture the gut microbiota and this relationship affects the health of the host. However, different analysis strategies can generate different views on the relative abundance of each microbial taxon, which can affect our conclusions about the significance of diet to gut health in lean and obese subjects. Here we explored the impact of using different analysis strategies to study the gut microbiota in a context of diet, health and obesity.

Methods. Over 15 million 16S rRNA gene sequences from published studies involving dietary interventions in obese laboratory rodents were analyzed. Three strategies were used to assign the 16S sequences to Operational Taxonomic Units (OTUs) based on the GreenGenes reference OTU sequence files clustered at $97 \%$ and $99 \%$ similarity.

Results. Different strategies to select OTUs influenced the relative abundance of all bacterial taxa, but the magnitude of this phenomenon showed a strong study effect. Different taxa showed up to $20 \%$ difference in relative abundance within the same study, depending on the analysis strategy. Very few OTUs were shared among the samples. ANOSIM test on unweighted UniFrac distances showed that study, sequencing technique, animal model, and dietary treatment (in that order) were the most important factors explaining the differences in bacterial communities. Except for obesity status, the contribution of diet and other factors to explain the variability in bacterial communities was lower when using weighted UniFrac distances. Predicted functional profile and high-level phenotypes of the microbiota showed that each study was associated with unique features and patterns.

Conclusions. The results confirm previous findings showing a strong study effect on gut microbial composition and raise concerns about the impact of analysis strategies on the membership and composition of the gut microbiota. This study may be helpful to guide future research aiming to investigate the relationship between diet, health, and the gut microbiota. 
1 Different analysis strategies of 16S rRNA gene data 2 from rodent studies generate contrasting views of gut 3 bacterial communities associated with diet, health and 4 obesity

Jose F. Garcia-Mazcorro ${ }^{1, *}$, Jorge R. Kawas ${ }^{2}$, Cuauhtemoc Licona-Cassani ${ }^{3}$, Susanne U. Mertens-Talcott ${ }^{4}$ and Giuliana Noratto ${ }^{5}$

${ }^{1}$ Research and Development, MNA de Mexico, San Nicolas de los Garza, Nuevo Leon, Mexico

${ }^{2}$ Faculty of Agronomy, Universidad Autonoma de Nuevo Leon, General Escobedo, Nuevo Leon, Mexico

${ }^{3}$ School of Engineering and Sciences, Tecnologico de Monterrey, Monterrey, Nuevo Leon, Mexico

${ }^{4}$ Department of Nutrition and Food Science, Texas A\&M University, College Station, Texas, USA

${ }^{5}$ Department of Food Science and Technology, Texas A\&M University, College Station, Texas, USA

Corresponding Author:

Jose Garcia-Mazcorro ${ }^{1}$

Avenida Acapulco 770, San Nicolas de los Garza, Nuevo Leon, 66477, Mexico

Email address: josegarcia_mex@hotmail.com

\section{Abstract}

Background. One of the main functions of diet is to nurture the gut microbiota and this relationship affects the health of the host. However, different analysis strategies can generate different views on the relative abundance of each microbial taxon, which can affect our conclusions about the significance of diet to gut health in lean and obese subjects. Here we explored the impact of using different analysis strategies to study the gut microbiota in a context of diet, health and obesity.

Methods. Over 15 million 16S rRNA gene sequences from published studies involving dietary interventions in obese laboratory rodents were analyzed. Three strategies were used to assign the 16S sequences to Operational Taxonomic Units (OTUs) based on the GreenGenes reference OTU sequence files clustered at $97 \%$ and $99 \%$ similarity.

Results. Different strategies to select OTUs influenced the relative abundance of all bacterial taxa, but the magnitude of this phenomenon showed a strong study effect. Different taxa showed up to $20 \%$ difference in relative abundance within the same study, depending on the analysis 
strategy. Very few OTUs were shared among the samples. ANOSIM test on unweighted UniFrac distances showed that study, sequencing technique, animal model, and dietary treatment (in that order) were the most important factors explaining the differences in bacterial communities. Except for obesity status, the contribution of diet and other factors to explain the variability in bacterial communities was lower when using weighted UniFrac distances. Predicted functional profile and high-level phenotypes of the microbiota showed that each study was associated with unique features and patterns.

Conclusions. The results confirm previous findings showing a strong study effect on gut microbial composition and raise concerns about the impact of analysis strategies on the membership and composition of the gut microbiota. This study may be helpful to guide future research aiming to investigate the relationship between diet, health, and the gut microbiota.

\section{Introduction}

The digestive tract of humans and other animals is inhabited by trillions of microbes and viruses that have evolved with their host as a unit throughout millennia. Host genetics is one of the most important factors shaping the gut microbiota (Bonder et al., 2016; Dabrowska \& Witkiewicz, 2016; Zhao et al., 2016; Knowles et al., 2019; Suzuki et al., 2019) but environmental factors may dominate over genetics in some circumstances (Muegge et al., 2011; Rothschild et al., 2018). Among all environmental factors that can modulate the gut microbiota, diet and dietary patterns have the strongest potential to do so. For example, diet can output similar microbiota functions across mammalian phylogeny (Muegge et al., 2011) and certain diets can induce and perpetuate obesity, a phenomenon that is closely interlinked with the gut microbiota (Bäckhed et al., 2004; Turnbaugh et al., 2008).

Several food ingredients such as polysaccharides and polyphenols have been reported to influence lipid metabolism by altering gut microbiota composition. However, there are still many unknowns about the effect of diet and other factors on the gut microbial ecosystem and host' health. For instance, microbes display high levels of cell-to-cell variability, even between members of the same strain under controlled homogeneous environments (Davidson et al., 2008). This individuality strengthens even more the notion of a highly personalized microbiome in each human or animal host, which ultimately affects host response to diet. Moreover, we often do not deal with the microbes per se; instead, we habitually deal with DNA nucleotide sequences obtained from these unique microbes at discrete time-points. In order to efficiently classify microbes based on molecular data (e.g. DNA sequences), scientists have developed categorization (or grouping) items and rules besides the idea of species, a term that is obviously ambiguous for organisms with eccentric reproductive strategies (Angert, 2005). The term Operational Taxonomic Unit (OTU) was invented in a context of numerical taxonomy (Sneath, 1957; Sokal \& Sneath, 1963) and nowadays is mostly used to catalogue genetic sequences from marker genes (e.g. the $16 \mathrm{~S}$ gene) based on nucleotide similarities. In short, similar sequences are catalogued within the same OTU, and therefore each OTU is thought to represent similar microorganisms. Alternatives to OTUs have been suggested (Callahan, McMurdie \& Holmes, 
2017) but the idea of using nucleotide similarities to catalogue microbes prevails. Note that the supposition that similar $16 \mathrm{~S}$ sequences come from similar organisms is far from being true (Jaspers et al., 2004).

Numerous studies have investigated the gut microbiota in relationship to diet, health and obesity. However, only few have looked at data from multiple studies to expose the impact of analysis strategies on this relationship. Lozupone et al. (2013) performed a meta-analysis of studies of the human microbiota (gut, oral, vaginal, skin and other) and showed that samples of the Western adult fecal microbiota clustered strongly by study. However, the authors did not discuss the potential contribution of diet in the differences in the microbiota and only used one approach to select OTUs. Walters et al. (2014) performed a similar meta-analysis of human gut microbes associated with obesity and Inflammatory Bowel Disease and showed that specific microbial signatures of obesity were not consistent between studies. The authors suggested that this was due to differences in effect sizes and explained that some conditions such as inflammatory bowel disease are associated with more obvious differences in microbiota compared to other conditions such as obesity, whose association with the microbiota, accordingly to the authors, is less clear. The authors of this study also did not look at the contribution of diet to the observed differences and did not explore the use of different analysis strategies. A recent meta-analysis of samples from rodents and humans confirmed that diet can induce reproducible microbiome alterations but only focused on high-fat diets and did not assess the impact of analysis strategies on the relative abundance of taxa (Bisanz et al., 2019). Others have used a different approach looking only at the -published-abundance of a few selected bacterial groups (e.g. Bifidobacterium) and their relationship with dietary components (So et al., 2018; Wilson et al., 2019).

The mucin-degrader bacterium Akkermansia muciniphila is a good example to emphasize the relevance of these issues. This bacterium has anti-inflammatory and anti-obesity effects that are mediated by a close biochemical interaction with the host through the colonic mucus (Cani \& de Vos, 2017). The relative abundance of this bacterium in feces ranges from $0.1 \%$ to up to $85 \%$, and this variation is often considered to reflect a response to dietary components and health status (Garcia-Mazcorro et al., 2020). However, some of this variation may also be derived from the specific analysis strategy used during the analysis, although this issue has received little attention (Garcia-Mazcorro et al., 2020). The aim of this study was to explore how different analysis strategies impact the results from gut microbiological studies in a context of diet, health and obesity.

\section{Materials \& Methods}

\section{Ethical Considerations}

This study used 16S rRNA gene sequence data from public datasets published by our research group (Table 1), all of which received approval for the use of the animals.

\section{$16 \mathrm{~S}$ gene sequencing data}


119 We used 16S gene sequencing data from seven of our previous publications dealing with 120 modulation of the gut microbiota using dietary interventions in animal obese models (Table 1).

121 Performing a comparative analysis of data generated by the same research group is advantageous 122 because technical variation is likely to be less compared to the variation obtained from multiple 123 research groups. The sequencing procedure was performed at UC Davis for three studies, while 124 the remaining four studies were sequenced at the Molecular Research LP. The Quick-DNA 125 Fecal/Soil Microbe Miniprep Kit (D6010, Zymo Research) was used in all studies with only 126 slight variations except for the peach study that used the QIAamp® PowerFecal® DNA kit 127 (Qiagen). We did not use FastPrep (MP Biomedicals) for bead beating in any of these studies 128 and this is important to clarify because some people consider the use of FastPrep essential for 129 optimal lysis.

Six factors were studied across the samples: 'dietary treatment', with 11 levels; 'study',

131

132

133

134

135

136

137

138

139

140

141

142

143

144

145

146

147

148

149

150

151

152

153

154

155

156

157

158

\section{Assignment of 16S sequences to OTUs}

Three strategies were used to assign the $16 \mathrm{~S}$ sequences to OTUs. First, the conservative closed approach that discards sequences for not matching any sequence in the sequence reference data. Second, a de novo approach, which does not use a reference data set to cluster the sequences (Westcott \& Schloss, 2015), thus being relatively free to depict the variety of sequences in a sample. Finally, an open approach that combines these two approaches (Rideout et al., 2014), first performing a closed approach followed by clustering of remaining sequences de novo. From this point on, we will refer to these strategies as the name of the clustering method (e.g. closed) and the reference file utilized (e.g. OTUs clustered at 97\%). For example, 'closed97' and 'closed99' will refer to a closed approach using the 97\% and the $99 \%$ OTU reference files, respectively. We only used the default $97 \%$ in the similarity option of the pick_otus.py script but changing this parameter can also drastically affect the results (more on this in "Similarity percentage between 16S rRNA gene sequences" in Supplemental Information).

\section{Chimeras}

Chimeras are possible combinations of two or more parent sequences that can inflate the observed OTUs and other diversity parameters, especially for de novo and open-reference 
159

160

161

162

163

164

165

166

167

168

169

170

171

172

173

174

175

176

177

178

179

180

181

182

183

184

185

186

187

188

189

190

191

192

193

194

195

196

197

strategies to select OTUs. However, there is a lack of consensus in chimera detection and removal and even the existence of true chimeras has been questioned. In this study, we subsampled the pynast alignment of representative sequences (one per OTU) separately for each study and applied the public version of uchime (v.4.2.40) available in Mothur for chimera detection. These analyses were performed on representative sequences from the de novo and open strategies. All the GreenGenes reference OTU sequence files (total: 14 files; OTUs clustered at different similarity percentages, from $99 \%$ similarity with 203,451 sequences, to $61 \%$ similarity with 22 sequences) were used to explore the behavior of uchime.

\section{Taxonomic and diversity analyses}

We combined all OTU tables (one for each of the seven studies) from the closed97 and the closed99 approaches into two separate OTU tables (one for each approach) and used these OTU tables for taxonomic classification and diversity analyses. The relative abundance of each taxon was calculated using all sequences (including possible chimeras and OTUs that appear only once) because it is impossible to detect true chimeras and the possible relevance of low abundant groups in the gut microbiome (Claussen et al., 2017). The unique fraction metric, or UniFrac, is a phylogenetic method for comparing microbial communities based on the phylogenetic distance between sets of taxa in a phylogenetic tree as the fraction of the branch length of the tree that leads to descendants from one environment or the other, but not both (Lozupone et al., 2005). Both weighted and unweighted UniFrac distances were used for comparing microbial communities because they can lead to different insights into factors that structure microbial communities (Lozupone et al., 2007). Principal Coordinate Analyses (PCoA) using these UniFrac distances were performed in QIIME and visualized using Emperor. Additionally, we used uniform manifold approximation and projection (UMAP, McInnes et al. 2018), a non-linear dimensionality reduction technique, to confirm the observed clusters, using the umap R package.

\section{Prediction of functional profiling and phenotypes}

PICRUSt (phylogenetic investigation of communities by reconstruction of unobserved states, Langille et al., 2013) was used to predict the functional profiling based on the $16 \mathrm{~S}$ sequences. PICRUSt results were analyzed in STAMP (Parks et al., 2010). Additionally, we used BugBase (Ward et al., 2017) to predict organism-level microbiome phenotypes for each study separately.

\section{QIIME2}

QIIME2 was introduced in 2017 on the basis of a plugin architecture that allows third parties to contribute functionality (Bolyen et al., 2019) and has been constantly updated ever since (more than 20 versions of QIIME2 have been released). This paper was conceived and started in QIIME1 in 2018 but because QIIME1 is no longer updated or supported, DADA2 (Callahan et al., 2016) and Deblur (Amir et al., 2017) were also used to select OTUs for a few selected studies in QIIME2, in part based on the observations made by Thompson et al. (2017) in a context of 
198

199

200

201

202

203

204

205

206

207

208

209

210

211

212

213

214

215

216

217

218

219

220

221

222

223

224

225

226

227

228

229

230

231

232

233

234

235

236

OTUs and sequence variants. One interesting feature of DADA2 is the removal of chimeric sequences.

\section{Statistical analysis}

The non-parametric analysis of similarities (ANOSIM) and the Adonis tests were used to determine whether the clustering of samples by a given factor (e.g. study) is statistically significant based on UniFrac distances, using the compare_categories.py script in QIIME with default number of permutations (999). In our experience, these tests usually have low sensitivity (they usually yield low $p$ values even for weak clustering of samples), therefore it is informative to look at both the $p$ values and the percentage of variation explained by the factor. We used the non-parametric Kruskal-Wallis to compare the number of OTUs between the different levels of any given factor, and also to compare the results from BugBase.

\section{Results}

We analyzed >15 million 16S reads from 164 samples from seven studies dealing with dietary interventions in obese laboratory animals (Table 1). In general, each study investigated the effect of different diets on the gut microbiota of obese laboratory animals and compared the results with data from control obese and/or lean animals (Supplemental Table S3).

We detected 5,246 OTUs using the combined OTU table from the closed97 approach $(n=162)$, and 8,898 OTUs from the closed99 approach $(n=163)$ (two samples from the closed97 and one sample from the closed99 approach with the lowest number of sequences were discarded to generate a better assessment of diversity). This difference of $\sim 3,600$ OTUs is substantial (70\% more OTUs compared to the closed97 approach) and likely reflects the higher number of reference OTUs available for clustering the unknown $16 \mathrm{~S}$ gene sequences. The number of detected OTUs from the closed97 approach was always lower, and with the exception of the peach study, the number of OTUs from the de novo approaches was always higher (Table 2). The percentage of possible chimeras in the OTU representative sequences ranged from $11 \%$ (wheat study) to 55\% (apple study) for the de novo approach (median: 21\%), and from 4\% (quinoa study) to $45 \%$ (apple study) for the open approach (median: 13\%). Interestingly, the percentage of possible chimeras reached a plateau in all the studies, where increasing the number of reference OTU sequences did no longer yield higher numbers of chimeras, and were lower for the open approach in all the studies with the exception of the raspberry study. No singletons (OTUs that appear only once) were found in any analysis using the open approach because the default script prevents it, but the de novo approach always showed $\sim 2$ times higher percentage of singletons compared to the closed approach, except for the peach and the cherry study (Table 2). The factor 'animal model' (mice and rats) generated the highest number of significantly different OTUs, and 'obesity status' (obese and lean) the lowest (Table 3). The analysis of the combined OTU tables from the closed97 and the closed99 approach showed that only 161 and 165 OTUs ( $\sim 3 \%$ of all OTUs detected) were present in $50 \%$ of the samples, respectively. Very few OTUs 
237 (3-4) were present in $>80 \%$ of samples using either approach, and no OTU was present in $>90 \%$ 238 of samples.

239

240 Community membership structure

241 Table 2 shows an accurate numerical impression of relative abundances of all bacterial phyla

242 across different OTU picking strategies, with up to $20 \%$ difference in relative abundance of some

243 taxa, depending on the strategy analysis. However, Table 2 lacks a general view of how each

244 taxon is represented across the different levels of each factor. A visual analysis of membership

245 data revealed interesting patterns, for instance, the relative abundances of Firmicutes and

246 Bacteroidetes were equally represented in each level of all six factors studied, while

247 Cyanobacteria was poorly represented in samples from mice (Figure 1). However, note that these

248 results may also be misleading when considering the very few OTUs that were shared across all

249 samples.

250

251

252

253

254

255

256

257

258

259

260

261

262

263

264

265

266

267

268

269

270

271

272

\section{Peach study}

The peach study was the only one using 454 pyrosequencing, a different DNA extraction method, and a different primer set. As expected, an increase in quality threshold reduced the number of sequences that passed quality filtering. This is important because it has been suggested that a more stringent quality filtering helps to "reduce the number of spurious OTUs" (Buza et al., 2019), although in practice it is difficult to determine the exact quality threshold to differentiate "true" vs "false" OTUs (Edgar, 2017). We will not discuss this issue for Illumina platforms because defaults have already been established (Bokulich et al., 2013).

Overall the different taxa remained similar in relative abundance but a higher base quality score (qual) threshold of 34 (default: qual 25) had a strong effect on the number of sequences available for OTU picking $(62,971$ sequences with qual 25 vs. 11,553 with qual 34) and consequently on the number of the OTUs discovered (758 OTUs with qual 25 vs 442 OTUs with qual 34 in the closed97 approach). The difference in qual threshold also had an effect in the proportions of Bacteroidetes that went down from $~ 37 \%$ (qual 25) to $15 \%$ (qual 34), and Firmicutes that went up from $\sim 50 \%$ to $70 \%$. This discrepancy was not related to the lower number of sequences available for OTU picking because a lower rarefaction in all other analyses (e.g. with qual 25) revealed similar relative proportions compared to the analysis with higher rarefaction depth. A higher base quality score threshold also affected the presence of some low abundant groups (e.g. at qual 34 Deferribacteres and Fusobacteria were not detected using all approaches).

In the peach study, Bacteroidetes displayed the highest standard deviation (SD) and showed the biggest difference $(\sim 5 \%)$ in relative abundances, particularly between the open 97 and

273

274

275 276 the open99 approaches (Table 2). The difference between the lowest and the highest value was minimal for Firmicutes ( $2 \%)$, Proteobacteria $(\sim 2 \%)$ and others (Table 2$)$. Tenericutes showed the highest $\mathrm{SD}$ /average ratio (68.7), which implies that the variability was proportionally higher in this taxon, and Firmicutes the lowest (1.7), which implies that the variability was 
277 proportionally lower in this taxon. The detected phyla varied from 10 (closed97) to 13 (de

278 novo97 and open97).

279

280 Wheat study

281 In the wheat study, Firmicutes displayed the highest SD and showed the biggest difference

$282(\sim 20 \%)$ in relative abundances, particularly between the closed97 and the closed99 approaches

283 (Table 2). This contrasts heavily with the biggest difference of $\sim 5 \%$ observed in the peach study.

284 This difference in closed approaches was also noticeable in Bacteroidetes ( 11\% difference),

285 Proteobacteria ( $\sim 10 \%$ difference), and others (Table 2$)$ and this was not related to rarefaction

286 depth. This difference was not noticeable for the open and the de novo approaches where

287 Firmicutes, Bacteroidetes, Proteobacteria and others showed very similar proportions in all cases

288 (Table 2). Verrucomicrobia showed the highest SD/average ratio (107.9) and Firmicutes the

289 lowest (11.3). The number of detected phyla varied between 10 (closed97) and 14 (de novo).

290

291 Quinoa study

292 Similar to the wheat study, in the quinoa study Firmicutes displayed the highest SD and showed 293 the biggest difference $(\sim 15 \%)$ in relative abundances, particularly in the closed approaches

294 (Table 2). This difference in closed approaches was also noticeable in Bacteroidetes $(\sim 11 \%$ 295 difference) and minimal for Proteobacteria ( $2 \%$ difference) and others, and this was again not 296 related to rarefaction depth. Also similar to the wheat study, this difference was not noticeable 297 for the open and the de novo approaches where Firmicutes, Bacteroidetes, Proteobacteria and 298 others showed very similar proportions in all cases (Table 2). Verrucomicrobia showed the 299 highest SD/average ratio (101.8) and Firmicutes the lowest (7.3). The number of detected phyla 300 varied from 8 (closed97) to 12 (de novo).

301

302

303

304

305

306

307

308

309

310

311

312

313

314

315

316

\section{Barley study}

In the barley study, Bacteroidetes displayed the highest SD but Firmicutes showed the biggest difference $(\sim 9 \%)$ in relative abundances, particularly in the closed approach (Table 2). This difference in closed approaches was minimal in Bacteroidetes ( $\sim 3 \%$ difference), Proteobacteria ( $\sim 2 \%$ difference) and others and this was again not related to rarefaction depth. Also similar to the other studies, this difference was not noticeable in the de novo and open approaches (Table 2). Verrucomicrobia showed the highest SD/average ratio (91.9) and Firmicutes the lowest (5.5). The number of detected phyla varied from 8 (closed97) to 12 (de novo).

\section{Cherry study}

In contrast to the wheat, quinoa and barley studies, where the closed97 and the closed99 approaches showed different abundance of taxa, in the cherry study there was good agreement between the closed 97 and the closed 99 approaches for Firmicutes, Bacteroidetes, Proteobacteria and other groups, but these approaches showed dissimilar proportions compared to the open and the de novo approaches (Table 2). The cherry study was also interesting because the de novo 
317 approach showed the presence of bacterial groups (e.g. Nitrospirae, Chlorobi, Planctomycetes)

318 that were not detectable using the closed and the open approaches, and this was likely not related

319 to rarefaction depth because the open approach used similar thresholds. Bacteroidetes displayed

320 the highest SD and showed the biggest difference $(\sim 7 \%)$ in relative abundances. Tenericutes

321 showed the highest SD/average ratio (100.9) and Firmicutes the lowest (3.4). The number of

322 detected phyla varied from 14 (open99) to 22 (de novo). The use of DADA2 and Deblur in

323 QIIME2 showed 1,329 and 1,263 OTUs, respectively. This is about half the lower number of

324 OTUs (2,439 with closed97) obtained with the other approaches (Table 2).

325

326

\section{Raspberry study}

327

Unlike the other studies discussed above, the raspberry study was very interesting because it had

328 the lowest variation among the different OTU picking approaches $(0.56 \%$ difference in

329

330

331

332

333

334

335

336

337

338

339

340

341

342

343

344

345

346

347

348

349

350

351

352

353

354

355

Bacteroidetes and $0.55 \%$ difference in Firmicutes between the lowest and the highest results), which was also reflected in the SD/average ratio (highest: 32.8 for Cyanobacteria, lowest: 1.2 for Firmicutes) (Table 2). Moreover, both the open 97 and the open99 approaches detected as many as 41 different taxa at the phylum level, while the number of taxa in all other studies using the same approach only varied from 8 to 22 . This strongly suggests that the microbiota data contained within each study is unique and may sometimes contain a high proportion of taxa that go unnoticed in other studies. This is very important in a context of the role of rare taxa in maintaining the stability of ecosystems (Jousset et al., 2017). DADA2 and Deblur showed 791 and 721 OTUs, respectively. This contrast with the numbers (2,751-92,486 OTUs) obtained from all approaches (Table 2).

\section{Apple study}

In the apple study, Bacteroidetes showed the highest variation and also the biggest difference ( $\sim 11 \%)$ especially between the closed and the de novo approaches (Table 2$)$. This difference between the two approaches was also noticeable in Firmicutes and Tenericutes (Table 2).

Tenericutes showed the highest SD/average ratio (77.3) and Proteobacteria the lowest (4.9). The number of detected phyla varied from 15 to 35, thus making the apple study the second study more variable after the raspberry study. The use of DADA2 and Deblur in QIIME2 showed 1,357 and 1,149 OTUs, respectively. This contrast with the numbers (2,095-153,681 OTUs) obtained from all approaches (Table 2).

\section{Firmicutes/Bacteroidetes ratio}

The first paper that discussed about the possible usefulness of the Firmicutes/Bacteroidetes ratio was published by Ley et al. (2005), where the showed that obese mice had $50 \%$ more Firmicutes, with a proportionally lower abundance of Bacteroidetes, thus leading to a higher Firmicutes/Bacteroidetes ratio compared to lean mice. However, each phylum is composed by hundreds of different species, and therefore the ratio between the two has little significance as 
356 discussed elsewhere (Delzenne \& Cani, 2011). In this current study, this ratio varied from 1.1 to

357

358

359

360

361

362

363

364

365

366

367

368

369

370

371

372

373

374

375

376

377

378

379

380

381

382

383

384

385

386

387

388

389

390

391

392

393

394

3.3 due to the differences in relative abundance in the two phyla (Table 2).

\section{The phylum Verrucomicrobia}

Firmicutes and Bacteroidetes are usually the most abundant phyla in the gut microbiota and they have attracted most of the attention. However, other taxa deserve attention to better comprehend the functioning of the gut microbial ecosystem. A. muciniphila is a taxon that has generated interest as a new generation probiotic candidate to help obese patients and is considered to be a member of Verrucomicrobia based on 16S gene analysis. Akkermansia was detected in $\sim 15 \%$ the samples $(25 / 164)$ with an average of $3 \%$ and it was more represented in colon samples (Figure 1). Interestingly, the whole Verrucomicrobia phylum also had an average of 3\%, which implies that most or all Verrucomicrobia was represented by Akkermansia. If we translate the proportions of $16 \mathrm{~S}$ reads into numbers, a 3\% would represent approximately 300 million cells $\left(3 \times 10^{8}\right)$ in a hypothetical environment of $1 \times 10^{10}$ cells/g of intestinal contents. While this conversion is not necessarily accurate, the high numbers may bear some ecological relevance if one considers that as low as 1,000 cells of other microbes are enough to thrive (Nilsson et al., 2019). In three studies, the abundance of Verrucomicrobia was $>5$ times higher using a closed approach compared to the other approaches (Table 2).

\section{The phylum Cyanobacteria}

Another group of interest for gut health is Cyanobacteria (Bárcena et al., 2019), which is often considered a contaminant and removed from 16S gene analysis. However, Ley et al. (2005) showed a deep-branching clade of Cyanobacteria in the guts of animals and mentioned that they may represent descendants of non-photosynthetic ancestral Cyanobacteria that have become adapted to life in the mammalian gut. Here, Cyanobacteria was detected in $\sim 50 \%$ the samples $(74 / 164)$ and was always low in relative abundance $(<0.5 \%)$ (Table 2$)$. While $0.5 \%$ may be considered low, this percentage implies approximately 50 million $\left(5 \times 10^{7}\right)$ cells in the same hypothetical environment of $1 \times 10^{10}$ cells/g of intestinal contents as discussed above.

\section{Analyses of UniFrac distances}

\section{UniFrac analyses from the closed97 approach}

The first two coordinates explained $18 \%$ of the variation using unweighted distances (Figure 2), which is similar to other studies that have shown that the first two coordinates explained $23 \%$ of the variation in a data set of unweighted UniFrac distances from samples of the human microbiota (Lozupone et al., 2013). Accordingly to ANOSIM tests on unweighted distances, study, sequencing procedure, animal model, and dietary treatment were the factors most highly associated with the differences in the bacterial communities (Figure 2, Table 4). UMAP confirmed the clustering by animal model and study (Supplemental Information). This is interesting because others have also shown a strong study effect (Lozupone et al., 2013). 
395

396

397

398

399

400

401

402

403

404

405

406

407

408

409

410

411

412

413

414

415

416

417

418

419

420

421

422

423

424

425

426

427

428

429

430

431

432

433

434

In this study, the first 2 coordinates explained $34 \%$ of the variation using the weighted UniFrac distances, which is about twice the variation explained by the first two axes using unweighted UniFrac, but produced a very similar clustering of samples. Accordingly to ANOSIM tests, the effect of study, animal model, and sequencing procedure explained much smaller proportion of the variability in the data using weighted distances, while obesity status explained a higher proportion of the variation $(23.6 \%$ vs $9.5 \%$ using unweighted distances, Table 4).

\section{UniFrac analyses from the closed99 approach}

The analysis of unweighted UniFrac distances from the closed99 approach revealed similar results compared to the closed97 regarding the relative contribution of each factor in explaining the variability in the data (Figure 3 and Table 5). The first two coordinates explained $25 \%$ of the variation (Figure 3), which is even closer to the results of Lozupone et al. (2013). Interestingly, Akkermansia was not part of the ten most abundant groups (Figure 3) and the factor 'study' explained most of the dissimilarity in the communities using the unweighted UniFrac data accordingly to the ANOSIM test (Table 5). In our experience it is uncommon that one single factor explains that much of the variability between microbial communities. Similar to the analysis using the closed97 approach, the clustering of samples was very similar but the effect of 'study', 'animal model' and 'sequencing procedure' at explaining the variability in the data was lower using weighted UniFrac, while obesity status explained much more of the variability in the data (Table 5).

\section{UniFrac analyses from closed97 approach on mice samples}

To discover any additional pattern or association between the microbial communities, we performed a separate analysis of mice samples only $(n=120)$. Briefly, unweighted UniFrac analyses using closed97 showed that 'treatment' and 'study' were the most important factors, each explaining almost half of the dissimilarity in the data accordingly to ANOSIM tests, while the use of weighted UniFrac distances revealed a lower contribution of 'study' and 'treatment' and a higher contribution of obesity status at differentiating microbial communities (more about this in "UniFrac analyses from closed97 approach on mice samples" in Supplemental Information).

\section{Prediction of functional profiling}

PICRUSt revealed a total of 217 features that were significantly different between different studies, 211 between different treatments, 145 between sequencing techniques, 136 between mice and rats, 69 between lean and obese, and 32 between feces and colon contents (adjusted $\mathrm{P}<$ 0.05, see 'PICRUSt results' in Supplemental Information). This is interesting because the factor 'study' was also associated with the highest amount of variability. The weighted Nearest Sequenced Taxon Index (weighted NSTI) score was developed to summarize the extent to which microorganisms in a given sample are related to sequenced genomes (e.g. a NSTI score of 0.03 
435 indicates that the genomes of the microbes were well represented and that the average microbe in 436 the sample can be predicted using a relative from the same "species"). Higher NSTI values 437 indicate a lower accuracy of the predictions. Here, NSTI scores varied widely, from a minimum 438 of 0.03 (sample W11, feces from lean mice sequenced with MiSeq, wheat study) to a maximum 439 of 0.34 (sample W32, feces from obese mice on quinoa sequences with MiSeq, quinoa study). 440 There was no indication to suggest that any group of sequences were associated with lower or

441

442

443

444

445

446

447

448

449

450

451

452

453

454

455

456

457

458

459

460

461

462

463

464

465

466

467

468

469

470

471

472

473

474 higher NSTI scores.

\section{Prediction of organism-level microbiome phenotypes}

BugBase revealed interesting differences in phenotypes within each study. For example, obese rats in a high-fat diet had higher proportions of bacteria with the potential of forming biofilms among the different treatments in the apple study, lean mice had higher proportions of bacteria containing mobile elements in the barley study, and obese mice had higher proportions of stresstolerant bacteria in the cherry study (more on this in 'BugBase results' in Supplemental Information). Overall, these results also indicate that each study showed unique peculiarities, ultimately derived from the molecular composition of the $16 \mathrm{~S}$ gene sequences at any given timepoint within each particular study.

\section{Discussion}

Bacteria and other microorganisms are artificially classified for various reasons, for example to understand their relationship with other organisms. In most scientific publications, it is implicitly assumed that this artificial classification yields biologically relevant groups that exist in nature at real, normal abundances in a state of "normobiosis". This cannot be better exemplified by the widespread use of the opposite term "dysbiosis", a term that is not only inaccurate but also misleading (Brüssow, 2020). However, the true abundance of any bacterial group in nature is very difficult to determine with accuracy, due to both biological facts (Davidson \& Surette, 2008; Jaspers \& Overmann, 2004), methodological biases, and the many remaining unknowns in microbiome research (Thomas \& Segata, 2019). This work provides clear evidence of this issue by showing that different analysis strategies generate up to $20 \%$ difference in relative abundance of some taxa from the same samples, depending on the strategy.

Our work also sheds light into the issue of the number of bacterial species in the gut. Considering the lowest sequence abundance threshold of one, it has been suggested that our planet harbor about 10,000 bacterial species (Caporaso et al., 2011) but others have suggested 5.6 million OTUs as the lower bound of the microbial diversity on Earth (Rideout et al., 2014). On the other hand, the human microbiome is thought to harbor 15,000 to 36,000 species (Frank et al., 2007) yet others have suggested only 4,930 species of bacteria in that environment (Pasolli et al., 2019). In technical terms, the number of OTUs is dependent on the number of sequences that pass quality filtering (i.e. the higher the quality threshold, the lower the number of sequences available to catalogue). However, the number of OTUs is also dependent on the specific strategy used to catalogue sequences into OTUs. For instance, in this study the number of OTUs from the 
475 closed97 approach was always lower, and with one exception, the number of OTUs from the de 476 novo approaches was always higher. While the higher number of OTUs detected by the de novo 477 approach may not be biologically relevant (Edgar, 2017), this may deserve more thought if we 478 consider the unexplored variety of microbes in nature.

479 As mentioned above, bacteria and other microbes display high levels of cell-to-cell 480 variability; in other words, these organisms show high levels of individuality, which implies that

481

482

483

484

485

486

487

488

489

490

491

492

493

494

495

496

497

498

499

500

501

502

503

504

505

506

507

508

509

510

511

512

513 every host carries a unique set of microbes. In support of this hypothesis, the analysis of the combined OTU tables from the closed 97 and the closed 99 approach showed that only very few OTUs were shared among the samples (3-4 OTUs were present in $>80 \%$ of samples using either approach, and no OTU was present in $>90 \%$ of samples). These results strongly suggest a unique microbial profile (i.e. variety of $16 \mathrm{~S}$ sequences) in each sample. More importantly, the fact that each bacterial cell displays a high level of individuality raises doubts about the relevance of nucleotide similarities in the 16S gene (or even whole genomes, see Lukjancenko, Wassenaar \& Ussery, 2010) to estimate or predict similarities in nature. In other words, it is feasible and very likely that even those same OTUs that were apparently shared by multiple samples represent in fact different microorganisms.

The analysis of $16 \mathrm{~S}$ data from each study revealed interesting patterns. However, there were two studies that attracted our attention. The raspberry study was very interesting because it had the lowest variation in relative abundance of taxa among the different OTU picking approaches, which was also reflected in the $\mathrm{SD} /$ average ratio. Also, in the raspberry study both the open97 and the open99 approaches detected as many as 41 different taxa at the phylum level, while the number of taxa in all other studies using the same approach only varied from 8 to 22 . This strongly suggests that the microbiota data contained within each study is unique and may sometimes contain a high proportion of taxa that go unnoticed in other studies. This is very important in a context of the role of rare taxa in maintaining the stability of ecosystems (Jousset et al., 2017).

This paper shows interesting results about the impact of the analysis strategies in the relative abundance of Verrucomicrobia and Cyanobacteria and these taxa are also important in a context of low abundant or rare taxa (or OTUs in datasets). Several strategies have been used to remove the so-called rare sequences. Needham et al. (2017) used 99\% de novo OTUs (i.e. OTUs generated using $99 \%$ similarity without the use of a reference database) that exceeded a threshold of $0.4 \%$ in relative abundance without providing any rationale for using this threshold. In contrast, Navas-Molina et al. (2013) filtered the data based on the proportions of sequences represented by the OTUs (the authors suggested to remove OTUs that represents less than $0.005 \%$ of all sequence reads based on the analyses by Bokulich et al., 2013) and Lozupone et al. (2013) discarded whole samples that did not have at least 100 sequences after quality filtering and OTU assignment. Although the decision of removing rare sequences is often based on rational arguments (Bokulich et al., 2013) it is still arbitrary to choose one strategy or another. This is important because the so-called rare microbes may in fact be keystone species that can 
514 regulate entire microbial environments, including host-associated microbiomes (Jousset et al., 515 2017).

516 The factor(s) that better explain the differences in gut microbial communities are of 517 interest for various disciplines in biomedical sciences. For instance, the results of this current

518 work showed that the factor 'animal model' generated the highest number of significantly 519 different OTUs, while 'obesity status' the lowest. Unlike other studies suggesting that microbial 520 signatures of obesity are not consistent between studies because of effect sizes (Walters et al., 521 2014), in this study the lack of statistical difference in gut microbiota between obese and lean is

522

523

524

525

526

527

528

529

530

531

532

533

534

535

536

537

538

539

540

541

542

543

544

545

546

547

548

549

550

551

552

553 most likely due to the fact that the obese rodents were subject to different dietary treatments, thus making difficult to dissect between obesity status and dietary treatment. In this regard, the results of the analysis of UniFrac distances are particularly important considering the thoughts discussed by Lozupone et al. (2007) that explained that unweighted UniFrac is better suited to detect effects of different "founding" populations (e.g. the source of bacteria that first colonize the gut of newborn individuals and factors that are restrictive for microbial growth such as temperature). In contrast, they suggested that weighted UniFrac can more accurately reveal the effects of more transient factors, for example nutrient availability. Host genetics can be considered as a founding effect that since the moment of birth imposes certain restrictions to growth of some microbes, and in our analyses the factor 'animal model' explained high proportion of the variation when using unweighted distances. In contrast, the analysis of weighted distances revealed that the effect of obesity status at sampling explained much more variability in the data compared to unweighted analyses, a result that also makes sense if we consider obesity as a more transient condition that suddenly appeared in the host-microbiota relationship. More research is needed to better investigate the relationship of the different factors as well as their interactions in the gut microbiota.

This paper is not free of limitations. For instance, the transport, storage and DNA extraction methods can drastically affect the reported microbial community structure (Martinez et al., 2019). These and other factors should be considered when studying the microbial composition of gut samples. Also, there is often overlap between factors affecting our view of the gut microbiota, for instance in this study the weight of the factor "sequencing technique" may be masked by the use of different primers or DNA extraction method, and obesity status influenced the dietary regimen (i.e. obese animals are more often subject to dietary manipulation). More importantly, the most recent ways to catalogue marker $16 \mathrm{~S}$ genes (e.g. DADA2) are offering more accurate but also more conservative views of microbial diversity that may need radical conceptual improvements, for example considering the evolution of the noncoding strand of the 16S rRNA gene (Garcia-Mazcorro \& Barcenas-Walls, 2016).

\section{Conclusions}

In summary, diet and the gut microbiota are strongly related to host health and their interactions are "convoluted and multi-faceted" ( $X u \&$ Knight, 2015). This paper aimed to contribute to current knowledge about the impact of using different analysis strategies in the relationship 
554 between the gut microbiota, diet and obesity. The results show that the use of different strategies

555 to select OTUs have an impact on the relative proportions of bacterial taxa, particularly when

556 using a closed OTU picking approach. This may be due to the higher number of reference OTUs

557 available for clustering the unknown $16 \mathrm{~S}$ gene sequences as well as the number of used reads

558 between closed97 and closed99 strategies. We also demonstrated the impact of using OTU

559 reference sequences clustered at different similarity percent and confirmed previous observations

560 regarding a strong study effect. We invite others to consider and further expand the feasible

561 possibility that the variations among studies are related to the individuality of bacteria. Overall,

562 the results are useful to guide future research and meta-analyses aiming to investigate the

563 complex relationship between diet, health, and the gut microbiota.

564

565

\section{Acknowledgements}

566

567

568

569

570

571

572

573

574

575

576

577

578

579

580

581

582

583

584

585

586

587

588

589

590

591

592

We thank all developers and users of QIIME, PICRUSt, STAMP, and BugBase, and especially to Jose Pablo Gomez from the Center for Animal Disease Modeling and Surveillance at UC Davis, for his valuable help with UMAP.

\section{References}

Amir A, McDonald D, Navas-Molina JA, Kopylova E, Morton JT, Xu ZZ, Kightley EP, Thompson LR, Hyde ER, Gonzalez A, Knight R. 2017. Deblur rapidly resolves singlenucleotide community sequence patterns. mSystems 2:e00191-16 DOI 10.1128/mSystems.00191-16.

Angert ER. 2005. Alternatives to binary fission in Bacteria. Nature Reviews Microbiology 3:214-224 DOI 10.1038/nrmicro1096.

Bäckhed F, Ding H, Wang T, Hooper LV, Young Koh G, Nagy A, Semenkovich CF, Gordon JI. 2004. The gut microbiota as an environmental factor that regulates fat storage. Proceedings of the Natural Academy of Sciences USA 101:15718-15723 DOI 10.1073/pnas.0407076101.

Bárcena C, Valdés-Mas R, Mayoral P, Garabaya C, Durand S, Rodríguez F, FernándezGarcía MT, Salazar N, Nogacka AM, Garatachea N, Bossut N, Aprahamian F, Lucia A, Kroemer G, Freije JMP, Quirós PM, López-Otín C. 2019. Healthspan and lifespan extension by fecal microbiota transplantation into progeroid mice. Nature Medicine 25:1234-1242. DOI 10.1038/s41591-019-0504-5.

Bisanz JE, Upadhyay V, Turnbaugh JA, Ly K, Turnbaugh PJ. 2019. Meta-analysis reveals reproducible gut microbiome alterations in response to a high-fat diet. Cell Host \& Microbe 26:265-272 DOI 10.1016/j.chom.2019.06.013.

Bonder MJ, Kurilshikov A, Tigchelaar EF, Mujagic Z, Imhann F, Vich Vila A, Deelen P, Vatanen T, Schirmer M, Smeekens SP, Zhernakova DV, Jankipersadsing SA, Jaeger M, Oosting M, Cenit MC, Masclee AAM, Swertz MA, Li Y, KKumar V, Joosten L, Harmsen H, Weersma RK, Franke L, Hofker MH, Xavier RJ, Jonkers D, 
593

594

595

596

597

598

599

600

601

602

603

604

605

606

607

608

609

610

611

612

613

614

615

616

617

618

619

620

621

622

623

624

625

626

627

628

629

630

631

Netea MG, Wijmenga C, Fu J, Zhernakova A. 2016. The effect of host genetics on the gut microbiome. Nature Genetics 48:1407-1412 DOI 10.1038/ng.3663.

Bokulich NA, Subramanian S, Faith JJ, Gevers D, Gordon JI, Knight R, Mills DA, Caporaso JG. 2013. Quality-filtering vastly improves diversity estimates from Illumina amplicon sequencing. Nature Methods 10:57-59 DOI 10.1038/nmeth.2276.

Bolyen E, Rideout JR, Dillon MR, Bokulich NA, Abnet CC, Al-Ghalith GA, Alexander H, Alm EJ, Arumugam M, Asnicar F, Bai Y, Bisanz JE, Bittinger K, Brejnrod A, Brislawn CJ, Titus Brown C, Callahan BJ, Caraballo-Rodríguez AM, Chase J, Cope EK, Da Silva R, Diener C, Dorrestein PC, Douglas GM, Durall DM, Duvallet C, Edwardson CF, Ernst M, Estaki M, Fouquier J, Gauglitz JM, Gibbons SM, Gibson DL, Gonzalez A, Gorlick K, Guo J, Hillmann B, Holmes S, Holste H, Huttenhower C, Huttley GA, Janssen S, Jarmusch AK, Jiang L, Kaehler BD, Bin Kang K, Keefe CR, Keim P, Kelley ST, Knights D, Koester I, Kosciolek T, Kreps J, Langille MGI, Lee J, Ley R, Liu Y-X, Loftfield E, Lozupone C, Maher M, Marotz C, Martin BD, McDonald D, McIver LJ, Melnik AV, Metcalf JL, Morgan SC, Morton JT, Turan Naimey A, Navas-Molina JA, Felix Nothias L, Orchanian SB, Pearson T, Peoples SL, Petras D, Lai Preuss M, Pruesse E, Buur Rasmussen L, Rivers A, Robeson II MS, Rosenthal P, Segata N, Shaffer M, Shiffer A, Sinha R, Jin Song S, Spear JR, Swafford AD, Thompson LR, Torres PJ, Trinh P, Tripathi A, Turnbaugh PJ, Ul-Hasan S, van der Hooft JJJ, Vargas F, Vázquez-Baeza Y, Vogtmann E, von Hippel M, Walters W, Wan Y, Wang M, Warren J, Weber KC, Williamson CHD, Willis AD, Xu ZZ, Zaneveld JR, Zhang Y, Zhu Q, Knight R, Caporaso JG. Reproducible, interactive, scalable and extensible microbiome data science using QIIME 2. Nature Biotechnology 37:852-857 DOI 10.1038/s41587-0190209-9.

Brüssow H. 2020. Problems with the concept of gut microbiota dysbiosis. Microbial Biotechnology 13:423-434 DOI 10.1111/1751-7915.13479.

Buza TM, Tonui T, Stomeo F, Tiambo C, Katani R, Schilling M, Lyimo B, Gwakisa P, Cattadori IM, Buza J, Kapur V. 2019. iMAP: an integrated bioinformatics and visualization pipeline for microbiome data analysis. BMC Bioinformatics 20:374 DOI 10.1186/s12859-019-2965-4.

Callahan BJ, McMurdie PJ, Holmes SP. 2017. Exact sequence variants should replace operational taxonomic units in marker-gene data analysis. The ISME Journal 11:26392643 DOI 10.1038/ismej.2017.119.

Callahan BJ, McMurdie PJ, Rosen MJ, Han AW, Johnson AJA, Holmes SP. 2016. DADA2: high-resolution sample inference from Illumina amplicon data. Nature Methods 13:581583 DOI 10.1038/nmeth.3869.

Cani PD, de Vos WM. 2017. Next-generation beneficial microbes: the case of Akkermansia muciniphila. Frontiers in Microbiology 8:1765 DOI 10.3389/fmicb.2017.01765. 
632

633

634

635

636

637

638

639

640

641

642

643

644

645

646

647

648

649

650

651

652

653

654

655

656

657

658

659

660

661

662

663

664

665

666

667

668

669

670

Caporaso JG, Kuczynski J, Stombaugh J, Bittinger K, Bushman FD, Costello EK, Fierer N, Gonzalez Peña A, Goodrich JK, Gordon JI, Huttley GA, Kelley ST, Knights D, Koenig JE, Ley RE, Lozupone CA, McDonald D, Muegge BD, Pirrung M, Reeder J, Sevinsky JR, Turnbaugh PJ, Walters WA, Widmann J, Yatsunenko T, Zaneveld J, Knight R. 2010. QIIME allows analysis of high-throughput community sequencing data. Nature Methods 7:335-336 DOI 10.1038/nmeth.f.303.

Caporaso JG, Lauber CL, Walters WA, Berg-Lyons D, Lozupone CA, Turnbaugh PJ, Fierer N, Knight R. 2011. Global patterns of $16 \mathrm{~S}$ rRNA diversity at a depth of millions of sequences per sample. Proceedings of the Natural Academy of Sciences USA 108:4516-4522 DOI 10.1073/pnas.1000080107.

Claussen JC, Skieceviĉienė J, Wang J, Rausch P, Karlsen TH, Lieb W, Baines JF, Franke A, Hütt MT. 2017. Boolean analysis reveals systematic interactions among lowabundance species in the human gut microbiome. PLoS Computational Biology 13:e1005361 DOI 10.1371/journal.pcbi.1005361.

Dabrowska K, Witkiewicz W. 2016. Correlations of host genetics and gut microbiome composition. Frontiers in Microbiology 7:1357 DOI 10.3389/fmicb.2016.01357.

Davidson CJ, Surette MG. 2008. Individuality in Bacteria. Annual Review of Genetics 42:253268 DOI 10.1146/annurev.genet.42.110807.091601.

Delzenne NM, Cani PD. 2011. Interaction between obesity and the gut microbiota: relevance in nutrition. Annual Review of Nutrition 31:15-31 DOI 10.1146/annurev-nutr-072610145146.

Edgar RC. 2017. Accuracy of microbial community diversity estimated by closed- and openreference OTUs. PeerJ 5:e3889 DOI 10.7717/peerj.3889.

Frank DN, St. Amand AL, Feldman RA, Boedeker EC, Harpaz N, Pace NR. 2007. Molecular-phylogenetic characterization of microbial community imbalances in human inflammatory bowel diseases. Proceedings of the Natural Academy of Sciences USA 104:13780-13785 DOI 10.1073/pnas.0706625104.

Garcia-Mazcorro JF, Barcenas-Walls JR. 2016. Thinking beside the box: Should we care about the non-coding strand of the 16S rRNA gene? FEMS Microbiology Letters 363:fnw171 DOI 10.1093/femsle/fnw171

Garcia-Mazcorro JF, Ivanov I, Mills DA, Noratto G. 2016. Influence of whole-wheat consumption on fecal microbial community structure of obese diabetic mice. PeerJ 4:e1702 DOI 10.7717/peerj.1702.

Garcia-Mazcorro JF, Lage NN, Mertens-Talcott S, Talcott S, Chew B, Dowd SE, Kawas JR, Noratto GD. 2018. Effect of dark sweet cherry powder consumption on the gut microbiota, short-chain fatty acids, and biomarkers of gut health in obese $\mathrm{db} / \mathrm{db}$ mice. PeerJ 6:e4195 DOI 10.7717/peerj.4195.

Garcia-Mazcorro JF, Mills DA, Murphy K, Noratto G. 2017. Effect of barley supplementation on the fecal microbiota, caecal biochemistry and key biomarkers of 
671

672

673

674

675

676

677

678

679

680

681

682

683

684

685

686

687

688

689

690

691

692

693

694

695

696

697

698

699

700

701

702

703

704

705

706

707

708

obesity and inflammation in obese $\mathrm{db} / \mathrm{db}$ mice. European Journal of Nutrition 57:25132528 DOI 10.1007/s00394-017-1523-y.

Garcia-Mazcorro JF, Mills DA, Noratto G. 2016. Molecular exploration of fecal microbiome in quinoa-supplemented obese mice. FEMS Microbiology Ecology 92:fiw089 DOI 10.1093/femsec/fiw089.

Garcia-Mazcorro JF, Minamoto Y, Kawas JR, Suchodolski JS, de Vos WM. 2020. Akkermansia and microbial degradation of mucus in cats and dogs: implications to the growing worldwide epidemic of pet obesity. Veterinary Sciences 7:44. DOI 10.3390/vetsci7020044.

Garcia-Mazcorro JF, Pedreschi R, Chew B, Dowd SE, Kawas JR, Noratto G. 2018. Dietary supplementation with raspberry extracts modifies the fecal microbiota in obese diabetic $\mathrm{db} / \mathrm{db}$ mice. Journal of Microbiology and Biotechnology 28:1247-1259 DOI 10.4014/jmb.1803.03020.

Garcia-Mazcorro JF, Pedreschi R, Yuan J, Kawas JR, Chew B, Dowd SE, Noratto G. 2019. Dietary supplementation with whole apples for six weeks is associated with a distinctive microbiota, proteomics and metabolomics profile in the gut of Dawley Sprague rats fed a high-fat diet. PLoS ONE 14:e0212586 DOI 10.1371/journal.pone.0212586.

Jaspers E, Overmann J. 2004. Ecological significance of microdiversity: identical 16S rRNA gene sequences can be found in Bacteria with highly divergent genomes and ecophysiologies. Applied Environmental Microbiology 70:4831-4839 DOI 10.1128/AEM.70.8.4831-4839.2004.

Jousset A, Bienhold C, Chatzinotas A, Gallien L, GObet A, Kurm V, Küsel K, Rilling MC, Rivett DW, Salles JF, van der Heijden MGA, Youssef NH, Zhang X, Wei Z, WH Gera Hol. 2017. Where less may be more: How the rare biosphere pulls ecosystems strings. The ISME Journal 11:853-862 DOI 10.1038/ismej.2016.174.

Knowles SCL, Eccles RM, Baltrūnaitė L. 2019. Species identity dominates over environment in shaping the microbiota of small mammals. Ecology Letters 22:826-837 DOI 10.1111/ele.13240.

Langille MGI, Zaneveld J, Caporaso JG, McDonald D, Knights D, Reyes JA, Clemente JC, Burkepile DE, Vega Thurber RL, Knight R, Beiko RG, Huttenhower C. 2013. Predictive functional profiling of microbial communities using 16S rRNA marker gene sequences. Nature Biotechnology 31:814-823 DOI 10.1038/nbt.2676.

Ley RE, Bäckhed F, Turnbaugh P, Lozupone CA, Knight RD, Gordon JI. 2005. Obesity alters gut microbial ecology. Proceedings of the Natural Academy of Sciences USA 102:11070-11075 DOI 10.1073/pnas.0504978102.

Lozupone CA, Stombaugh J, Gonzalez A, Ackermann G, Wendel D, Vázquez-Baeza Y, Jansson JK, Gordon JI, Knight R. 2013. Meta-analyses of studies of the human microbiota. Genome Research 23:1704-1714. DOI 10.1101/gr.151803.112.

PeerJ reviewing PDF | (2020:06:50081:2:0:NEW 19 Oct 2020) 
709

710

711

712

713

714

715

716

717

718

719

720

721

722

723

724

725

726

727

728

729

730

731

732

733

734

735

736

737

738

739

740

741

742

743

744

745

746

747

Lozupone C, Knight R. 2005. UniFrac: a new phylogenetic method for comparing microbial communities. Applied Environmental Microbiology 71:8228-8235 DOI 10.1128/AEM.71.12.8228-8235.2005.

Lozupone CA, Hamady M, Kelley ST, Knight R. 2007. Quantitative and qualitative $\beta$ diversity measures lead to different insights into factors that structure microbial communities. Applied Environmental Microbiology 73:1576-1585 DOI 10.1128/AEM.01996-06.

Lukjancenko O, Wassenaar TM, Ussery DW. 2010. Comparison of 61 sequenced Escherichia coli genomes. Microbial Ecology 60:708-720 DOI 10.1007/s00248-010-9717-3.

Martínez N, Hidalgo-Cantabrana C, Delgado S, Margolles A, Sánchez B. 2019. Filling the gap between collection, transport and storage of the human gut microbiota. Scientific Reports 9:8327 DOI 10.1038/s41598-019-44888-8.

McInnes L, Healy J, Saul N, Grossberger L. 2018. UMAP: Uniform Manifold Approximation and Projection. Journal of Open Source Software 3:861. DOI 10.21105/joss.00861.

Muegge BD, Kuczynski J, Knights D, Clemente JC, González A, Fontana L, Henrissat B, Knight R, Gordon JI. 2011. Diet drives convergence in gut microbiome functions across mammalian phylogeny and within humans. Science 332:970-974. DOI 10.1126/science.1198719.

Navas-Molina JA, Peralta-Sanchez JM, Gonzalez A, McMurdie PJ, Vázquez-Baeza Y, Xu Z, Ursell LK, Lauber C, Zhou H, Song SJ, Huntley J, Ackermann GL, Berg-Lyons D, Holmes S, Caporaso JG, Knight R. 2013. Advancing our understanding of the human microbiome using QIIME. Methods Enzymology 531:371-444 DOI 10.1016/B978-0-12-407863-5.00019-8.

Needham DM, Sachdeva R, Fuhrman JA. 2017. Ecological dynamics and co-occurrence among marine phytoplankton, bacteria and myoviruses shows microdiversity matters. The ISME Journal 11:1614-1629 DOI 10.1038/ismej.2017.29.

Nilsson OR, Kari L, Steele-Mortimer O. 2019. Foodborne infection of mice with Salmonella Typhimurium. PLoS ONE 14:e0215190 DOI 10.1371/journal.pone.0215190.

Noratto GD, Garcia-Mazcorro JF, Markel M, Martino HS, Minamoto Y, Steiner JM, Byrne D, Suchodolski JS, Mertens-Talcott SU. 2014. Carbohydrate-free peach (Prunus persica) and plum (Prunus salicina) juice affects fecal microbial ecology in an obese animal model. PLoS ONE 9:e101723 DOI 10.1371/journal.pone.0101723.

Parks DH, Beiko RG. 2010. Identifying biologically relevant differences between metagenomic communities. Bioinformatics 26:715-721 DOI 10.1093/bioinformatics/btq041.

Pasolli E, Asnicar F, Manara S, Zolfo M, Karcher N, Armanini F, Beghini F, Manghi P, Tett A, Ghensi P, Collado MC, Rice BL, DuLong C, Morgan XC, Golden CD, Quince C, Huttenhower C, Segata N. 2019. Extensive unexplored human microbiome diversity revealed by over 150,000 genomes from metagenomes spanning age, geography, and lifestyle. Cell 176:649-662 DOI 10.1016/j.cell.2019.01.001.

Peer) reviewing PDF | (2020:06:50081:2:0:NEW 19 Oct 2020) 
748

749

750

751

752

753

754

755

756

757

758

759

760

761

762

763

764

765

766

767

768

769

770

771

772

773

774

775

776

777

778

779

780

781

782

783

784

785

786

787

Rideout JR, He Y, Navas-Molina JA, Walters WA, Ursell LK, Gibbons SM, Chase J, McDonald D, Gonzalez A, Robbins-Pianka A, Clemente JC, Gilbert JA, Huse SM, Zhou H-W, Knight R, Caporaso JG. 2014. Subsampled open-reference clustering creates consistent, comprehensive OTU definitions and scales to billions of sequences. PeerJ 2:e545 DOI 10.7717/peerj.545.

Rothschild D, Weissbrod O, Barkan E, Kurilshikov A, Korem T, Zeevi D, Costea PI, Godneva A, Kalka IN, Bar N, Shilo S, Lador D, Vich Vila A, Zmora N, PevsnerFisher M, Israeli D, Kosower N, Malka G, Chen Wolf B, Avnit.Sagi T, LotanPompan M, Weinberger A, Halpern Z, Carmi S, Fu J, Wijmenga C, Zhernakova A, Elinav E, Segal E. 2018. Environment dominates over host genetics in shaping human gut microbiota. Nature 555:210-215 DOI 10.1038/nature25973.

Sneath PHA. 1957. The application of computers to taxonomy. Journal of General Microbiology 17:201-226.

So D, Whelan K, Rossi M, Morrison M, Holtmann G, Kelly JT, Shanahan ER, Staudacher HM, Campbell KL. 2018. Dietary fiber intervention on gut microbiota composition in healthy adults: a systematic review and meta-analysis. The American Journal of Clinical Nutrition 107:965-983 DOI 10.1093/ajen/nqy041.

Sokal RR, Sneath PHA. Principles of numerical taxonomy. San Francisco: W.H. Freeman, 1963.

Suzuki TA, Phifer-Rixey M, Mack KL, Sheehan MJ, Lin D, Bi K, Nachman MW. 2019. Host genetic determinants of the gut microbiota of wild mice. Molecular Ecology 28:3197-3207 DOI 10.1111/mec.15139.

Thomas AM, Segata N. 2019. Multiple levels of the unknown in microbiome research. BMC Biology 17:48. DOI 10.1186/s12915-019-0667-z.

Thompson LR, Sanders JG, McDonald D, Amir A, Ladau J, Locey KJ, Prill RJ, Tripathi A, Gibbons SM, Ackermann G, Navas-Molina JA, Janssen S, Kopylova E, VázquezBaeza Y, González A, Morton JT, Mirarab S, Xu ZZ, Jian L, Haroon MF, Kanbar J, Zhu Q, Song SJ, Kosciolek T, Bokulich NA, Lefler J, Brislawn CJ, Humphrey G, Owens SM, Hampton-Marcell J, Berg-Lyons D, McKenzie V, Fierer N, Fuhrman JA, Clauset A, Stevens RL, Shade A, Pollard KS, Goodwin KD, Jansson JK, Gilbert JA, Knight R, The Earth Microbiome Project Consortium. 2017. A communal catalogue reveals Earth's multiscale microbial diversity. Nature 551:457-463 DOI 10.1038 /nature24621.

Turnbaugh PJ, Bäckhed F, Fulton L, Gordon JI. 2008. Diet-induced obesity is linked to marked but reversible alterations in the mouse distal gut microbiome. Cell Host \& Microbe 3:213-223 DOI 10.1016/j.chom.2008.02.015.

Walters WA, Xu Z, Knight R. 2014. Meta-analyses of human gut microbes associated with obesity and IBD. FEBS Letters 588:4223-4233 DOI 10.1016/j.febslet.2014.09.039.

Ward T, Larson J, Meulemans J, Hillmann B, Lynch J, Sidiropoulos D, Spear JR, Caporaso G, Blekhman R, Knight R, Fink R, Knights D. 2017. BugBase predicts organism-level microbiome phenotypes. bioRxiv DOI 10.1101/133462.

Peer) reviewing PDF | (2020:06:50081:2:0:NEW 19 Oct 2020) 
788 Westcott SL, Schloss PD. 2015. De novo clustering methods outperform reference-based

789

790

791

792

793

794

795

796

797

798

799 methods for assigning 16S rRNA gene sequences to operational taxonomic units. PeerJ 3:e1487 DOI 10.7717/peerj.1487.

Wilson B, Rossi M, Dimidi E, Whelan K. 2019. Prebiotics in irritable bowel syndrome and other functional bowel disorders in adults: a systematic review and meta-analysis of randomized controlled trials. American Journal of Clinical Nutrition 109:1098-1111. DOI 10.1093/ajen/nqy376.

Xu Z, Knight R. 2015. Dietary effects on human gut microbiome diversity. British Journal of Nutrition 113:S1-S5 DOI 10.1017/S0007114514004127.

Zhao P, Irwin DM, Dong D. 2016. Host genetics is associated with the gut microbial community membership rather than the structure. Molecular Biosystems 12:1676-1686 DOI 10.1039/C5MB00850F. 


\section{Figure 1}

Proportions of relative abundances (percentages) of $16 \mathrm{~S}$ reads.

The data is shown accordingly to the factors (A) animal model, (B) obesity status, (C) sequencing, (D) anatomical site, (E) study, and (F) treatment. Letters in bars represent different taxa (A: Firmicutes, B: Bacteroidetes, C: Proteobacteria, D: Verrucomicrobia, E:

Deferribacteres, F: Actinobacteria, G: Cyanobacteria, and H: others). The bars do not represent the relative abundance of each taxon; instead, they show the proportion of $16 \mathrm{~S}$ relative abundances from each level of the factors investigated. A balanced stacked bar denotes that the taxon was equally represented in each level of the factor (e.g. Firmicutes and Bacteroidetes between levels of all factors). An unbalanced stacked bar denotes that the taxon was more or less represented between the levels of the factor, for example Cyanobacteria between mice and rats, or between colon and feces. 

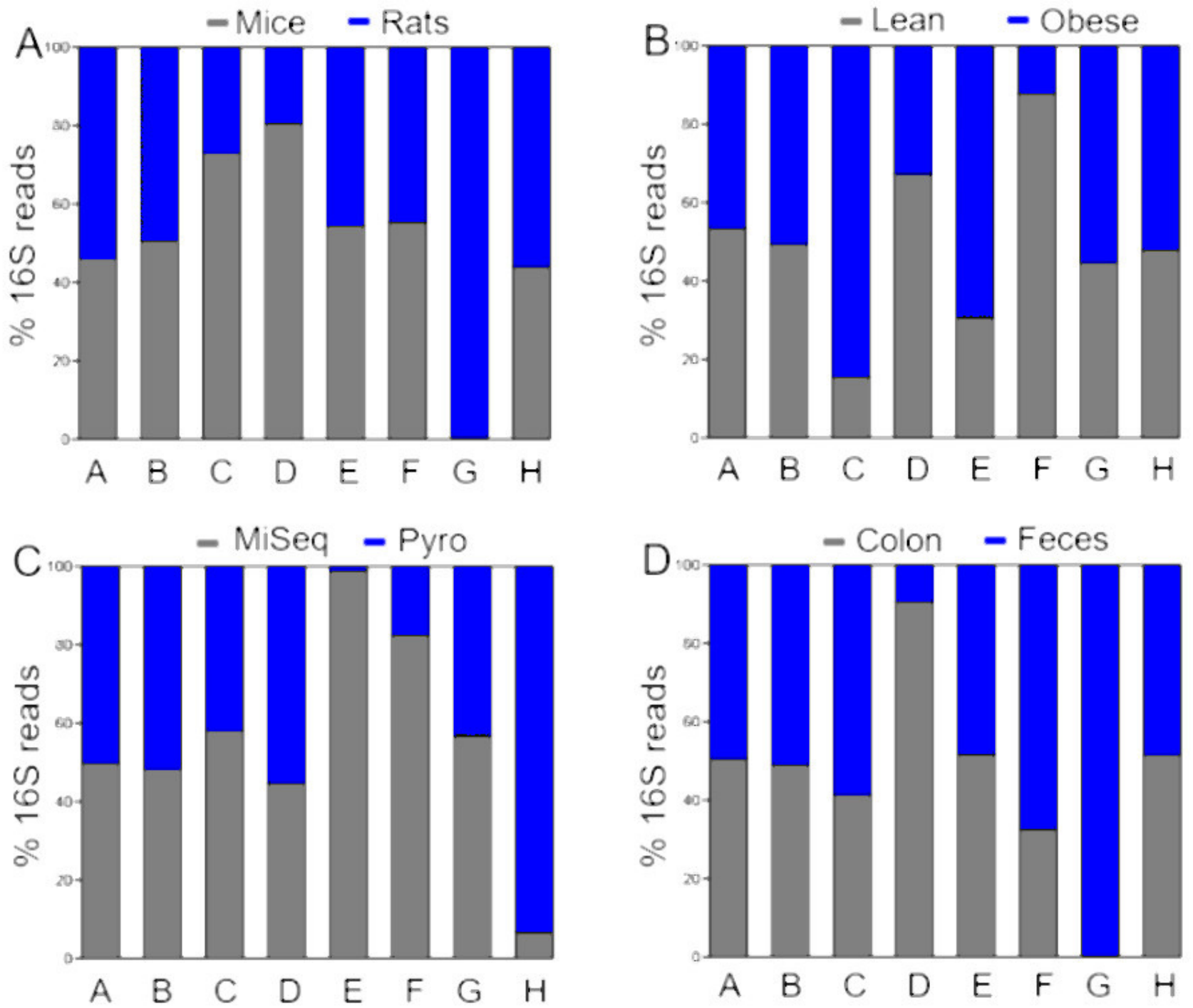

-Apple -Barley -Cherry -Peach

- Barley -Cherry -Lean -Obese -Quinoa - Raspberry -Wheat

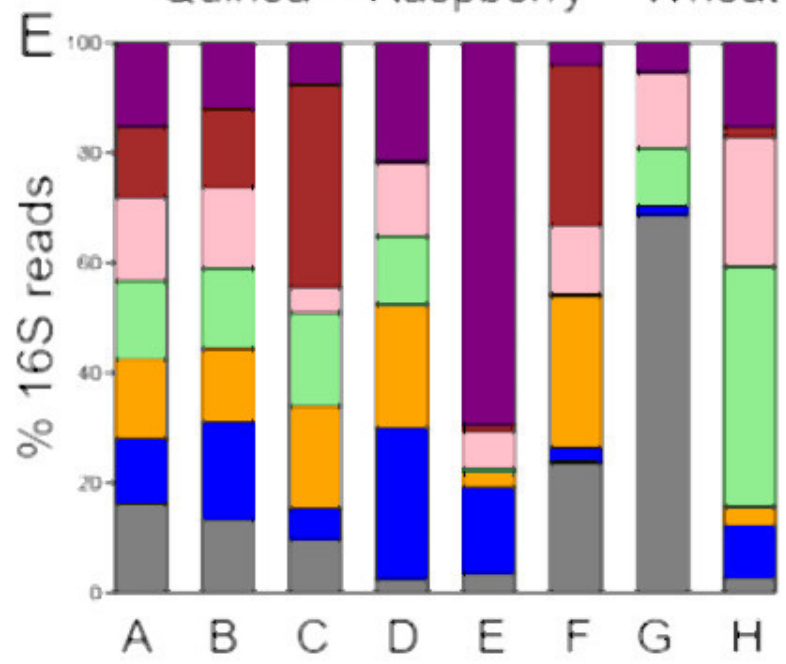
- HF apple - LF apple - Peach - Plum

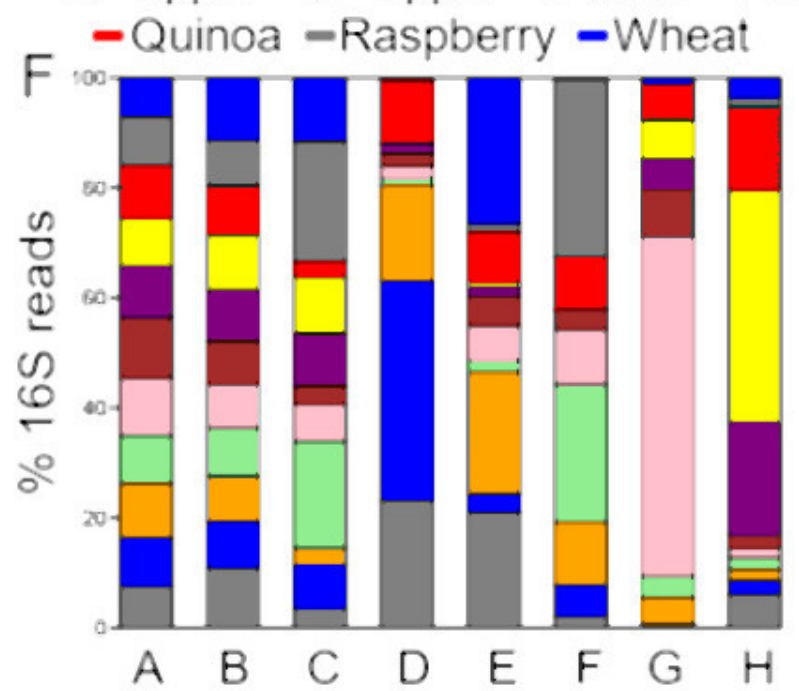




\section{Figure 2}

PCoA plots of unweighted UniFrac distances using data from the closed approach using the reference OTUs sequence file at $97 \%$ similarity (closed97 approach).

Each point represents a sample from one of the studies detailed in Table 1, and the plots highlight the effect of (A) animal model, (B) obesity status, (C) sequencing technique, (D) anatomical site, (E) study and (F) treatment. The labels "lean" and "obese" refer to lean and obese controls. The ten most abundant bacterial groups are superimposed in all plots (the bigger the circle, the bigger the relative abundance of each taxa) and labelled with numbers on the first plot (1: Ruminococcaceae, 2: Bacteroides, 3: Clostridiales, 4: Lachnospiraceae, 5: Oscillospira, 6: Enterobacteriaceae, 7: S24-7, 8: Lactobacillus, 9: Akkermansia, 10: Allobaculum) to show that clustering of samples is driven by specific bacterial groups that have previously been shown to influence (or be influenced by) health status, such as Akkermansia. The values for each axis are only shown in A to facilitate viewing. These plots were built using a rarefaction depth of 100 sequences per sample to account for as many samples as possible (only two samples were left out using this rarefaction depth). The UniFrac data was obtained from the closed97 approach but other approaches may reveal other patterns (see Table 4 and Table 5). 

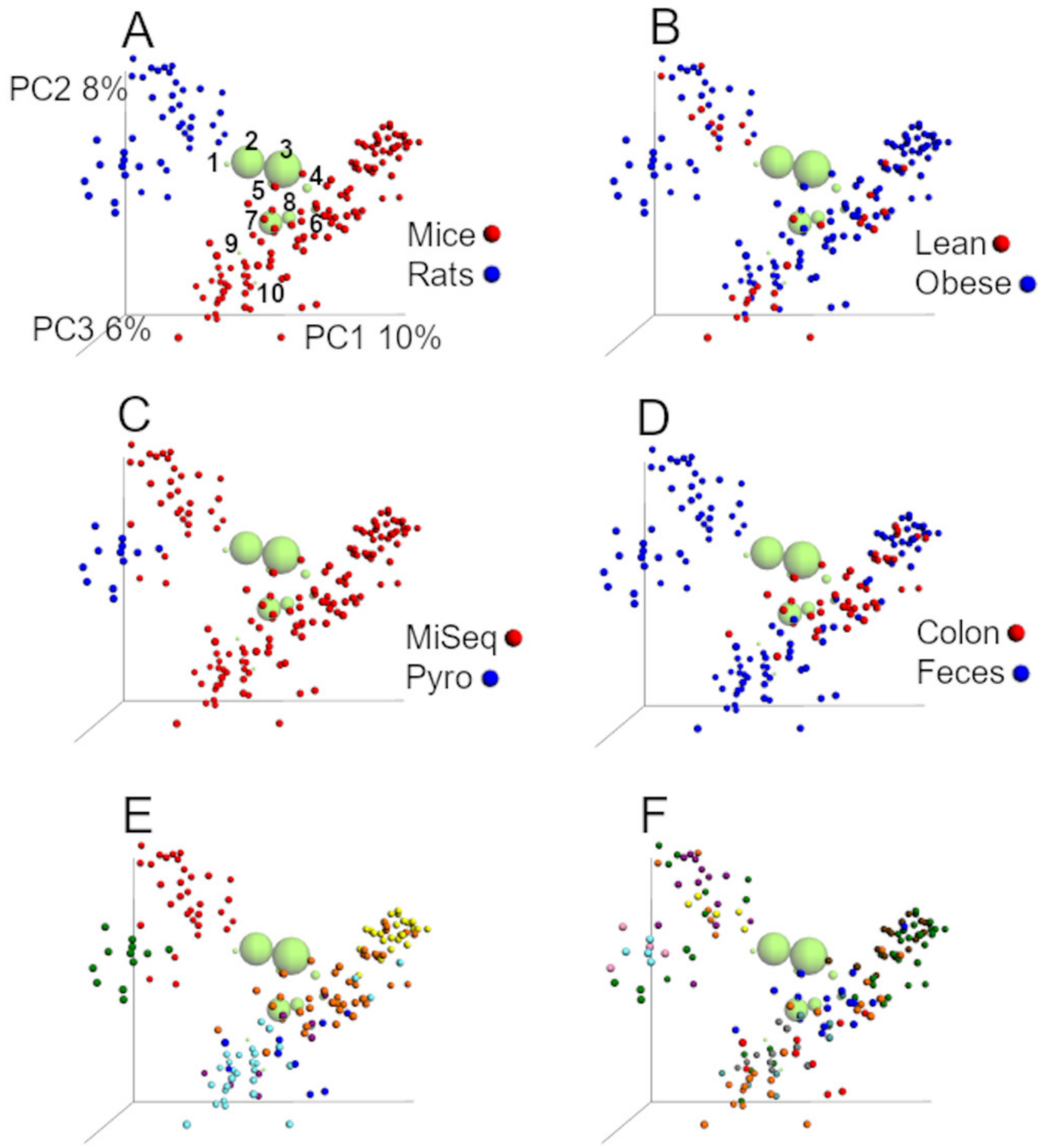

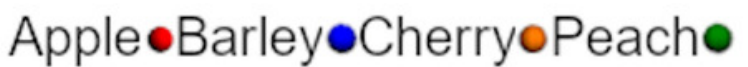
Quinoa $\bullet$ Raspberry $\bullet$ Wheato

Barley $\bullet$ Cherry $\bullet$ Lean $\bullet$ Obese $\bullet \mathrm{HF}$ apple $\bullet$ LF appleoPeachoPlumoQuinoa॰ Raspberry• Wheat $\bullet$ 


\section{Figure 3}

PCoA plots of unweighted UniFrac distances using data from the closed approach using the reference OTUs sequence file at $99 \%$ similarity (closed99 approach).

Each point represents a sample from one of the studies detailed in Table 1, and the plots highlight the effect of (A) animal model, (B) obesity status, (C) sequencing technique, (D) anatomical site, (E) study and (F) treatment. The labels "lean" and "obese" refer to lean and obese controls. The ten most abundant bacterial groups are superimposed in all plots (the bigger the circle, the bigger the relative abundance of each taxa) and labelled with numbers on the first plot (1: Ruminococcaceae, 2: Bacteroides, 3: Clostridiales, 4: Lachnospiraceae, 5: Oscillospira, 6: Enterobacteriaceae, 7: S24-7, 8: Lactobacillus, 9: Parabacteroides, 10: Allobaculum) to show that clustering of samples is driven by specific bacterial groups that have previously been shown to influence (or be influenced by) health status, such as Ruminococcaceae. The values for each axis are only shown in A to facilitate viewing. These plots were built using a rarefaction depth of 440 sequences per sample to account for as many samples as possible (only two samples were left out using this rarefaction depth). The Unifrac data was obtained from the closed99 approach but other approaches may reveal other patterns (see Table 4 and Table 5). 

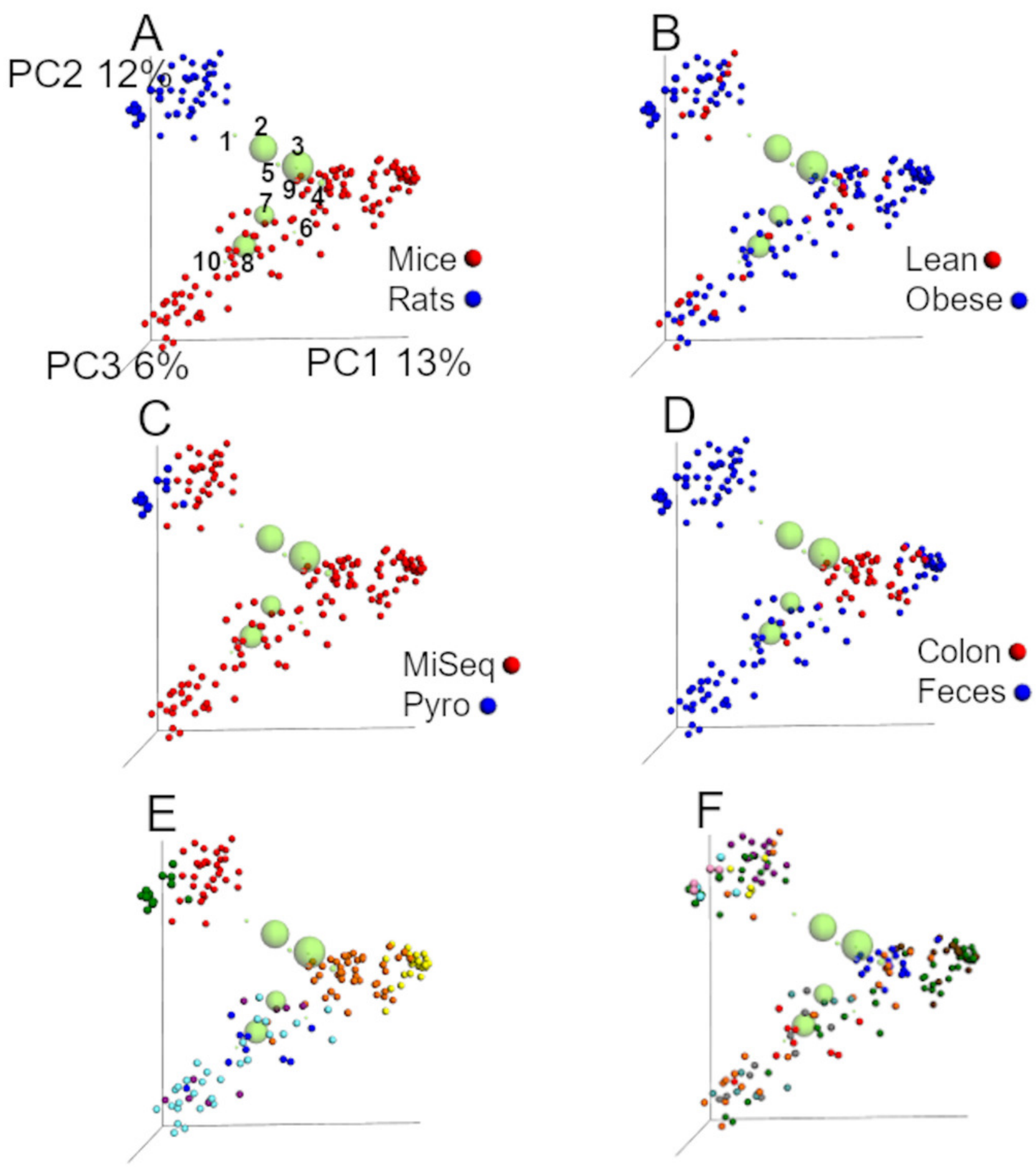

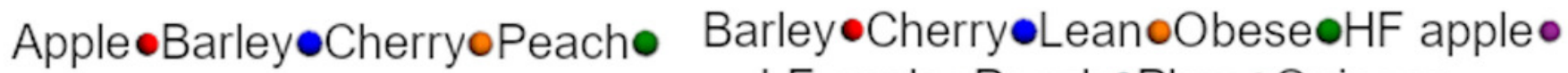
Quinoa॰Raspberry॰Wheato 


\section{Table 1 (on next page)}

List of publications from which the data for this study came from. 


\begin{tabular}{|c|c|c|c|c|c|}
\hline Publication & $\begin{array}{l}\text { Number of } \\
\text { samples }\end{array}$ & SRA Bioproject & Animal species & Sequencing Technique & Reported OTUs \\
\hline $\begin{array}{l}\text { Peach (Noratto et al. } \\
2014 \text { ) }\end{array}$ & 12 & PRJNA217444 & Zucker rats (obese) & Pyrosequencing & $\begin{array}{l}1,549 \text { (approach not } \\
\text { mentioned) }\end{array}$ \\
\hline $\begin{array}{l}\text { Wheat (Garcia- } \\
\text { Mazcorro et al. } \\
\text { 2016) }\end{array}$ & 30 & PRJNA281761 & $\mathrm{db} / \mathrm{db}$ mice & MiSeq (Illumina) & 8,686 (open)/1,302 (closed) \\
\hline $\begin{array}{l}\text { Quinoa (Garcia- } \\
\text { Mazcorro, Mills \& } \\
\text { Noratto 2016) }\end{array}$ & $9^{\mathrm{a}}$ & PRJNA299688 & $\mathrm{db} / \mathrm{db}$ mice & MiSeq & 5,774 (open) \\
\hline $\begin{array}{l}\text { Barley (Garcia- } \\
\text { Mazcorro et al. } \\
\text { 2017) }\end{array}$ & $10^{\mathrm{a}}$ & PRJNA314690 & $\mathrm{db} / \mathrm{db}$ mice & MiSeq & 5,366 (open) \\
\hline $\begin{array}{l}\text { Cherry (Garcia- } \\
\text { Mazcorro et al. } \\
\text { 2018) }\end{array}$ & $44^{\mathrm{b}}$ & PRJNA407462 & $\begin{array}{l}\mathrm{db} / \mathrm{db} \text { mice (colon } \\
\text { microbiota) }\end{array}$ & MiSeq & Not mentioned $^{\mathrm{c}}$ \\
\hline $\begin{array}{l}\text { Raspberry (Garcia- } \\
\text { Mazcorro et al. } \\
2018 \text { ) }\end{array}$ & 27 & PRJNA415476 & $\mathrm{db} / \mathrm{db}$ mice & MiSeq & $675^{\mathrm{d}}$ (open) \\
\hline $\begin{array}{l}\text { Apple (Garcia- } \\
\text { Mazcorro et al. } \\
\text { 2019) }\end{array}$ & 32 & PRJNA504388 & Dawley Sprague rats & MiSeq & $69,010\left(1,339^{\mathrm{d}}\right)$ (open) \\
\hline \multicolumn{6}{|c|}{ 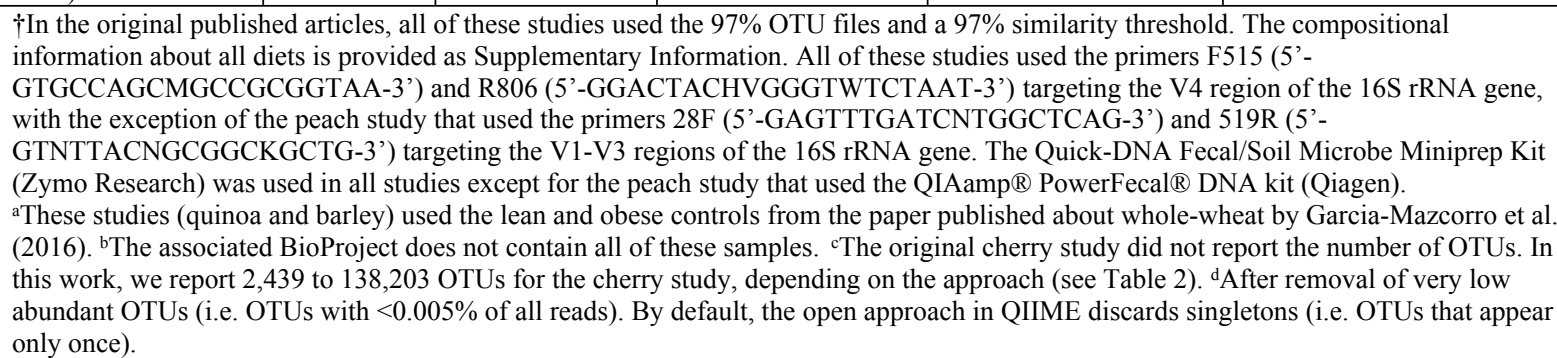 } \\
\hline
\end{tabular}




\section{Table 2 (on next page)}

Number of OTUs and relative proportions (i.e. percentages of 16S rRNA gene sequences) of the most abundant phyla. 


\begin{tabular}{|c|c|c|c|c|c|c|c|c|c|}
\hline & & & & \multicolumn{3}{|c|}{ Peach study } & & \multirow[b]{2}{*}{ Tenericutes } & \multirow{2}{*}{ Cyanobacteria } \\
\hline Approach & OTUs & $\begin{array}{l}\text { Singletons } \\
\end{array}$ & Firmicutes & Bacteroidetes & Proteobacteria & Verrucomicrobia & Actinobacteria & & \\
\hline Closed97 & $758 \downarrow$ & $114(15 \%)$ & $50.39 \%$ & $40.84 \% \uparrow$ & $6.09 \% \uparrow$ & $2.37 \% \uparrow$ & $0.072 \% \uparrow$ & $0.128 \% \downarrow$ & $0.065 \% \downarrow$ \\
\hline Closed99 & 1,074 & $192(18 \%)$ & $52.35 \% \uparrow$ & $39.31 \%$ & $5.77 \%$ & $2.23 \%$ & $0.069 \%$ & $0.148 \%$ & $0.077 \%$ \\
\hline De novo & 1,549 & $147(9 \%)$ & $50.14 \% \downarrow$ & $35.95 \%$ & $4.28 \% \downarrow$ & $1.67 \% \downarrow$ & $0.042 \%$ & $1.766 \%$ & $0.112 \%$ \\
\hline Open97 & 1,603 & NA & $50.18 \%$ & $35.15 \% \downarrow$ & $4.29 \%$ & $1.73 \%$ & $0.040 \% \downarrow$ & $1.766 \%$ & $0.113 \% \uparrow$ \\
\hline Open99 & $1,680 \uparrow$ & $\mathrm{NA}$ & $50.15 \%$ & $39.95 \%$ & $4.29 \%$ & $1.73 \%$ & $0.042 \%$ & $1.775 \% \uparrow$ & $0.112 \%$ \\
\hline \multicolumn{3}{|r|}{ SD: } & 0.88 & $2.45 \uparrow$ & 0.85 & 0.31 & $0.02 \downarrow$ & 0.85 & 0.02 \\
\hline & & & & \multicolumn{3}{|c|}{ Wheat study } & & & \\
\hline Approach & OTUs & Singletons & Firmicutes & Bacteroidetes & Proteobacteria & Verrucomicrobia & Actinobacteria & Tenericutes & Cyanobacteria \\
\hline Closed97 & $1,302 \downarrow$ & $414(32 \%)$ & $45.81 \% \downarrow$ & $32.52 \%$ & $13.41 \% \uparrow$ & $5.89 \% \uparrow$ & $2.25 \% \downarrow$ & $0.0249 \%$ & $0.0282 \% \downarrow$ \\
\hline Closed99 & 2,008 & $734(37 \%)$ & $65.97 \% \uparrow$ & $21.29 \% \downarrow$ & $7.64 \%$ & $2.06 \%$ & $2.90 \%$ & $0.0161 \% \downarrow$ & $0.0456 \%$ \\
\hline De novo & $37,474 \uparrow$ & $28,466(76 \%)$ & $57.96 \%$ & $31.82 \%$ & $3.18 \% \downarrow$ & $0.81 \% \downarrow$ & $3.46 \%$ & $0.3405 \%$ & $0.0927 \%$ \\
\hline Open97 & 8,686 & NA & $58.73 \%$ & $32.78 \%$ & $3.22 \%$ & $0.85 \%$ & $3.53 \% \uparrow$ & $0.3471 \%$ & $0.0912 \%$ \\
\hline Open99 & 9,013 & $\mathrm{NA}$ & $58.73 \%$ & $32.79 \% \uparrow$ & $3.21 \%$ & $0.85 \%$ & $3.53 \% \uparrow$ & $0.3476 \% \uparrow$ & $0.0908 \%$ \\
\hline \multirow{2}{*}{\multicolumn{4}{|c|}{ SD: }} & 4.53 & 4.19 & 2.03 & 0.52 & 0.17 & $0.03 \downarrow$ \\
\hline & & & & \multicolumn{3}{|c|}{ Quinoa study } & & & \\
\hline Approach & OTUs & Singletons & Firmicutes & Bacteroidetes & Proteobacteria & Verrucomicrobia & Actinobacteria & Tenericutes & Cyanobacteria \\
\hline Closed97 & $1,062 \downarrow$ & $333(31 \%)$ & $55.34 \% \downarrow$ & $30.29 \% \uparrow$ & $4.68 \% \uparrow$ & $7.20 \% \uparrow$ & $2.20 \% \downarrow$ & $0.051 \%$ & $0.050 \%$ \\
\hline Closed99 & 1,606 & $578(36 \%)$ & $69.77 \% \uparrow$ & $21.32 \% \downarrow$ & $2.73 \%$ & $3.29 \%$ & $2.61 \%$ & $0.045 \% \downarrow$ & $0.070 \% \uparrow$ \\
\hline De novo & $17,046 \uparrow$ & $11,500(67 \%)$ & $63.70 \%$ & $27.53 \%$ & $2.45 \% \downarrow$ & $1.04 \% \downarrow$ & $3.30 \%$ & $0.280 \% \uparrow$ & $0.041 \%$ \\
\hline Open97 & 5,774 & NA & $64.08 \%$ & $28.18 \%$ & $2.46 \%$ & $1.07 \%$ & $3.35 \%$ & $0.275 \%$ & $0.037 \%$ \\
\hline Open99 & 5,976 & NA & $64.07 \%$ & $28.17 \%$ & $2.46 \%$ & $1.07 \%$ & $3.36 \% \uparrow$ & $0.275 \%$ & $0.035 \% \downarrow$ \\
\hline \multirow{2}{*}{\multicolumn{4}{|c|}{$4.62 \uparrow$}} & 3.04 & 0.89 & 2.49 & 0.49 & 0.12 & $0.01 \downarrow$ \\
\hline & & & & \multicolumn{3}{|c|}{ Barley study } & & & \\
\hline Approach & OTUs & Singletons & Firmicutes & Bacteroidetes & Proteobacteria & Verrucomicrobia & Actinobacteria & Tenericutes & Cyanobacteria \\
\hline Closed97 & $1,078 \downarrow$ & $342(32 \%)$ & $53.50 \% \downarrow$ & $32.38 \%$ & $5.09 \% \uparrow$ & $6.63 \% \uparrow$ & $2.29 \% \downarrow$ & $0.0157 \%$ & $0.022 \% \downarrow$ \\
\hline $\begin{array}{ll}\text { Closed99 } \\
\end{array}$ & 1,586 & $572(36 \%)$ & $62.54 \% \uparrow$ & $29.04 \% \downarrow$ & $3.01 \%$ & $2.67 \%$ & $2.60 \%$ & $0.0131 \% \downarrow$ & $0.053 \%$ \\
\hline De novo & $15,599 \uparrow$ & $10,429(67 \%)$ & $55.65 \%$ & $36.03 \%$ & $2.08 \%$ & $1.20 \% \downarrow$ & $3.25 \%$ & $0.2859 \%$ & $0.106 \% \uparrow$ \\
\hline Open97 & 5,366 & NA & $55.86 \%$ & $36.75 \%$ & $2.07 \%$ & $1.23 \%$ & $3.32 \%$ & $0.2809 \%$ & $0.104 \%$ \\
\hline Open99 & 5,594 & $\mathrm{NA}$ & $55.85 \%$ & $36.77 \% \uparrow$ & $2.06 \% \downarrow$ & $1.23 \%$ & $3.32 \% \uparrow$ & $0.2812 \%$ & $0.102 \%$ \\
\hline \multirow{2}{*}{\multicolumn{4}{|c|}{ SD: }} & $3.14 \uparrow$ & 1.21 & 2.17 & 0.45 & 0.14 & $0.04 \downarrow$ \\
\hline & & & & \multicolumn{3}{|c|}{ Cherry study } & & & \\
\hline Approach & OTUs & Singletons & Firmicutes & Bacteroidetes & Proteobacteria & \begin{tabular}{|l|} 
Verrucomicrobia \\
\end{tabular} & Actinobacteria & Tenericutes & Cyanobacteria \\
\hline Closed97 & $2,439 \downarrow$ & $1,942(80 \%)$ & $50.89 \%$ & $36.60 \%$ & $6.75 \% \uparrow$ & $4.67 \%$ & $0.180 \%$ & $0.000 \% \downarrow$ & $0.000 \% \downarrow$ \\
\hline Closed99 & 4,217 & $3,226(77 \%)$ & $51.68 \% \uparrow$ & $36.00 \% \downarrow$ & $6.18 \%$ & $5.11 \% \uparrow$ & $0.172 \% \downarrow$ & $0.012 \%$ & $0.001 \%$ \\
\hline De novo & $138,203 \uparrow$ & $64,000(46 \%)$ & $47.27 \% \downarrow$ & $43.20 \% \uparrow$ & $4.80 \%$ & $3.46 \%$ & $0.208 \%$ & $0.079 \%$ & $0.002 \%$ \\
\hline Open97 & 69,658 & NA & $48.53 \%$ & $41.71 \%$ & $4.73 \% \downarrow$ & $2.90 \% \downarrow$ & $0.208 \%$ & $0.096 \% \uparrow$ & $0.002 \% \uparrow$ \\
\hline Open99 & 70,886 & NA & $48.53 \%$ & $41.75 \%$ & $4.73 \% \downarrow$ & $2.90 \% \downarrow$ & $0.209 \% \uparrow$ & $0.001 \%$ & $0.002 \%$ \\
\hline \multicolumn{3}{|r|}{ SD: } & 1.68 & $3.12 \uparrow$ & 0.90 & 0.98 & 0.02 & 0.05 & $0.00 \downarrow$ \\
\hline & & & & \multicolumn{3}{|c|}{ Raspberry study } & & & \\
\hline Approach & OTUs & Singletons & Firmicutes & Bacteroidetes & Proteobacteria & Verrucomicrobia & Actinobacteria & Tenericutes & Cyanobacteria \\
\hline Closed97 & $2,751 \downarrow$ & $722(26 \%)$ & $44.99 \% \downarrow$ & $40.66 \% \uparrow$ & $13.33 \% \uparrow$ & $0.052 \% \uparrow$ & $0.092 \%$ & $0.002 \% \downarrow$ & $0.001 \% \downarrow$ \\
\hline Closed99 & 4,433 & $1,231(28 \%)$ & $45.13 \%$ & $40.53 \%$ & $13.32 \%$ & $0.052 \% \uparrow$ & $0.092 \%$ & $0.002 \% \downarrow$ & $0.001 \% \downarrow$ \\
\hline De novo & $92,486 \uparrow$ & $69,306(75 \%)$ & $46.13 \% \uparrow$ & $39.40 \% \downarrow$ & $12.90 \% \downarrow$ & $0.049 \% \downarrow$ & $0.097 \% \uparrow$ & $0.005 \% \uparrow$ & $0.002 \% \uparrow$ \\
\hline Open97 & 21,243 & NA & $46.10 \%$ & $39.73 \%$ & $12.99 \%$ & $0.049 \% \downarrow$ & $0.088 \% \downarrow$ & $0.004 \%$ & $0.001 \% \downarrow$ \\
\hline \multirow[t]{3}{*}{ Open99 } & 21,834 & $\mathrm{NA}$ & $46.10 \%$ & $39.72 \%$ & $12.99 \%$ & $0.049 \% \downarrow$ & $0.088 \% \downarrow$ & $0.004 \%$ & $0.001 \% \downarrow$ \\
\hline & & & 0.55 & $0.56 \uparrow$ & 0.19 & 0.002 & 0.004 & 0.001 & $0.0004 \downarrow$ \\
\hline & & & & \multicolumn{3}{|c|}{ Apple study } & & & \\
\hline Approach & OTUs & Singletons & Firmicutes & Bacteroidetes & Proteobacteria & Verrucomicrobia & Actinobacteria & Tenericutes & Cyanobacteria \\
\hline Closed97 & $2,095 \downarrow$ & $510(24 \%)$ & $55.32 \% \downarrow$ & $39.17 \% \uparrow$ & $3.48 \% \downarrow$ & $0.499 \%$ & $0.270 \%$ & $0.002 \% \downarrow$ & $0.542 \% \uparrow$ \\
\hline Closed99 & 3,363 & $907(27 \%)$ & $55.43 \%$ & $38.74 \%$ & $3.97 \% \uparrow$ & $0.510 \% \uparrow$ & $0.246 \% \downarrow$ & $0.002 \% \downarrow$ & $0.448 \%$ \\
\hline De novo & $153,681 \uparrow$ & $81,032(53 \%)$ & $61.48 \%$ & $28.63 \% \downarrow$ & $3.89 \%$ & $0.279 \% \downarrow$ & $0.432 \%$ & $1.517 \%$ & $0.361 \%$ \\
\hline Open97 & 69,010 & NA & $61.84 \% \uparrow$ & $29.28 \%$ & $3.96 \%$ & $0.282 \%$ & $0.435 \%$ & $1.578 \% \uparrow$ & $0.364 \%$ \\
\hline \multirow[t]{2}{*}{ Open99 } & 70,056 & NA & $61.83 \%$ & $29.61 \%$ & $3.96 \%$ & $0.282 \%$ & $0.436 \%$ & $1.577 \%$ & $0.364 \%$ \\
\hline & & & 3.25 & $5.14 \uparrow$ & 0.19 & 0.12 & 0.09 & 0.79 & $0.07 \downarrow$ \\
\hline & & & & & & & & & $\begin{array}{l}\text { refer to the } \\
\text { these results } \\
\text { uences" in } \\
\text { his). SD: }\end{array}$ \\
\hline
\end{tabular}




\section{Table 3(on next page)}

Number of OTUs that were significantly different accordingly to the different factors. 
Table 3 Number of OTUs that were significantly different* accordingly to the different factors.

\begin{tabular}{|l|c|c|}
\hline Factor & FDR & Bonferroni \\
\hline Study (7 levels) & 309 & 174 \\
\hline Treatment (11 levels) & 278 & 105 \\
\hline Sequencing (two levels) & 225 & 112 \\
\hline Animal model (two levels) & 134 & 53 \\
\hline Anatomical site (two levels) & 31 & 14 \\
\hline Obesity (two levels) & 4 & 3 \\
\hline
\end{tabular}

*Defined as an adjusted $\mathrm{p}<0.01$ in non-parametric Kruskal-Wallis (adjusted for False Discovery Rate and Bonferroni). 


\section{Table 4 (on next page)}

Summary of results for all samples $(n=162)$ from the Adonis and ANOSIM tests for comparing categories using UniFrac data from the closed97 approach. 


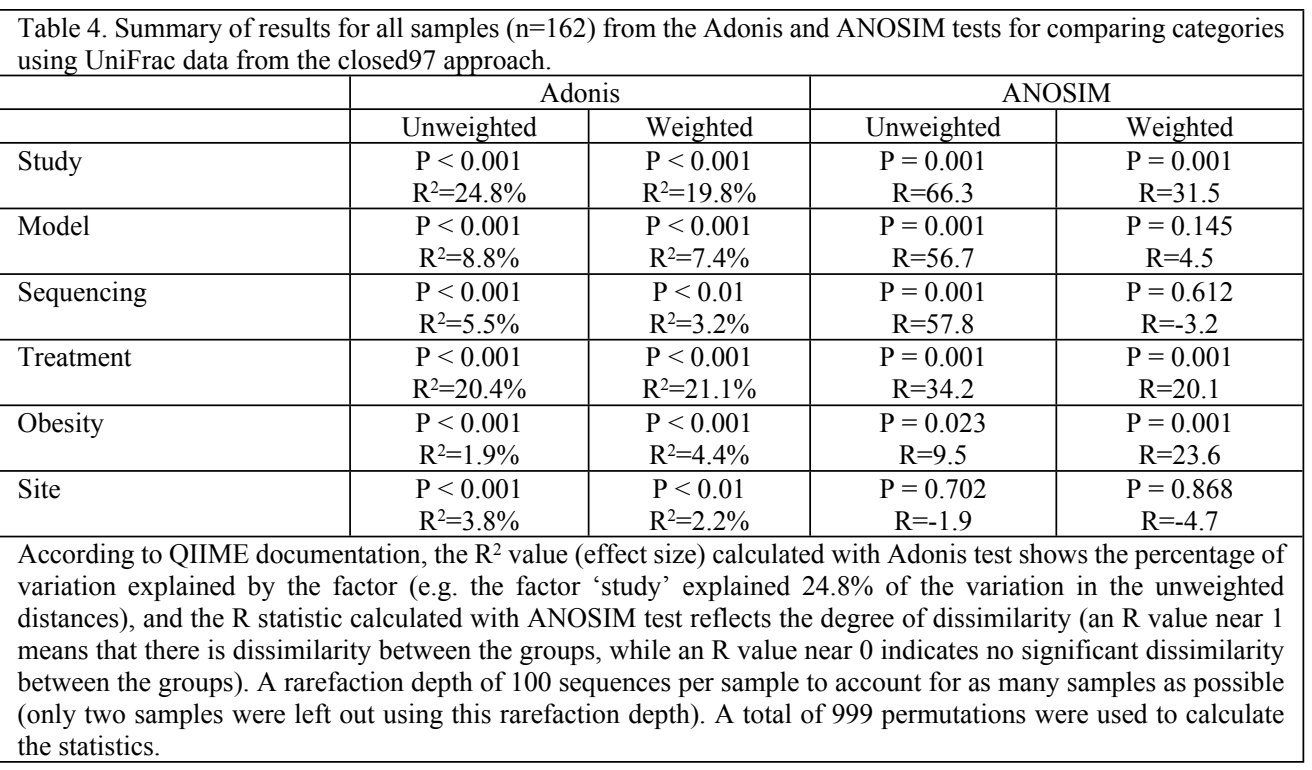

1 


\section{Table 5 (on next page)}

Summary of results for all samples $(n=163)$ from the Adonis and ANOSIM tests for comparing categories using UniFrac data from the closed99 approach. 


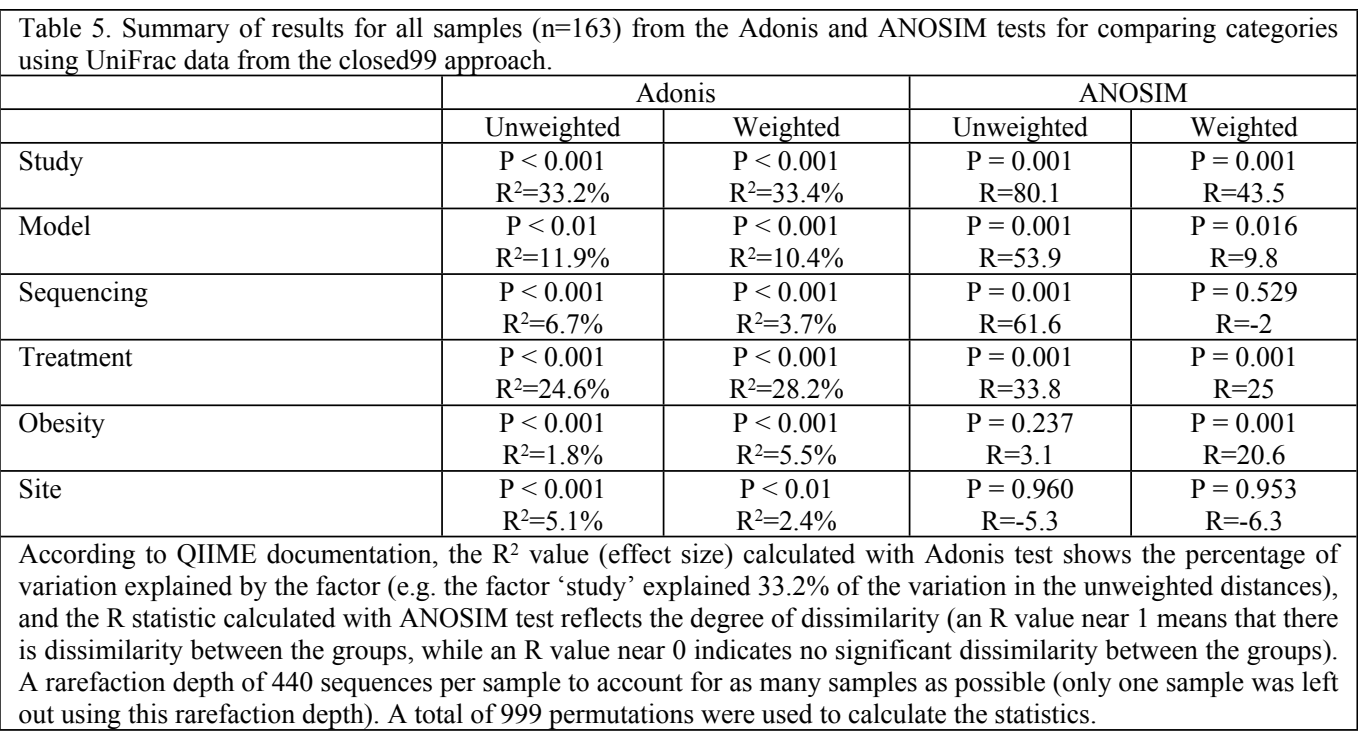

1 